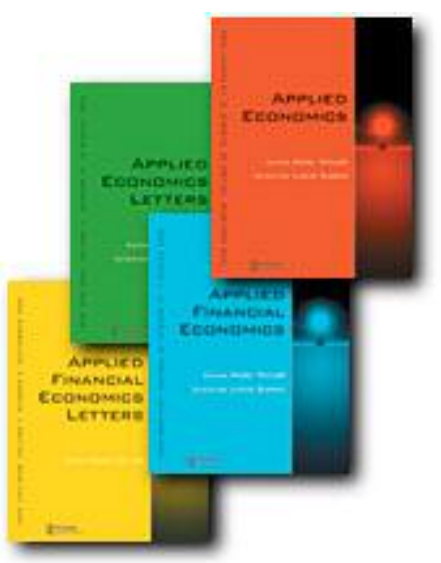

PRICE CONVERGENCE IN THE EUROPEAN CAR MARKET

\begin{tabular}{|r|l|}
\hline Journal: & Applied Economics \\
\hline Manuscript ID: & APE-05-0727.R1 \\
\hline Journal Selection: & Applied Economics \\
\hline JEL Code: & $\begin{array}{l}\text { F15 - Economic Integration < F1 - Trade < F - International } \\
\text { Economics }\end{array}$ \\
\hline Keywords: & $\begin{array}{l}\text { Market integration, Automobiles, Euroepan Union, Euro, Exchange } \\
\text { rates }\end{array}$ \\
\hline
\end{tabular}

powered by ScholarOne

Manuscript Central 


\title{
PRICE CONVERGENCE IN THE EUROPEAN CAR MARKET
}

\author{
Salvador Gil-Pareja* \\ (Universidad de Valencia) \\ Simón Sosvilla-Rivero \\ (FEDEA and Universidad Complutense de Madrid)
}

Version: November 2005

\begin{abstract}
This paper examines price convergence in the European Union car market over the period 1995-2005. The results indicate that there is a clear evidence of price convergence among the EU15 countries, but not before 1999. Moreover, countries of the Economic and Monetary Union started convergence previously to the EU15 as a whole. Finally, exchange rate changes has significantly contributed to price dispersion over time across countries.
\end{abstract}

JEL classification numbers: F15; F30.

Key Words: Market integration, Automobiles, European Union, Euro, Exchange rates.

\footnotetext{
"Corresponding author: Salvador Gil Pareja, Departamento de Estructura Económica, Av. de los Naranjos s/n, Valencia CP 46022, Spain; email: Salvador.Gil-Pareja@uv.es. We would like to thank José Antonio Martínez Serrano and Oscar Bajo Rubio for valuable comments and suggestions. We also thank Frank Verboven and Pinelopi Goldberg for sending us the price data in Denmark, Finland and Greece prior to 1999. Salvador Gil-Pareja acknowledges financial support from Ministerio de Ciencia y Tecnología (project SEC2003-05836/ECO). Simón Sosvilla-Rivero gratefully acknowledges the financial support by Ministerio de Ciencia y Tecnología grant SEJ2005-09094/ECON.
} 


\section{Introduction}

Over the past decades, important steps have been taken in the European Union (EU) to integrate markets. One of the expected effects of the process of market integration in Europe is price convergence. This hope relies on the argument that the elimination of administrative and technical barriers to trade, as a result of the Single Market Project, and the recent adoption of the euro reduce the potential for price discrimination across member States by bolstering cross-border trade and price transparency.

There is a growing number of papers on the issues of price and inflation convergence among the EU member States (see, e. g., Camarero et al., 2000; Rogers, 2001; Rogers et al., 2001; Gámez-Amián and Morales-Zumaquero, 2002; SosvillaRivero and Gil-Pareja, 2004; Chen, 2004; or Gil-Pareja and Sosvilla-Rivero, 2004) and the Economic and Monetary Union (EMU) countries (see, e. g., Parsley and Wei, 2001; Rogers, 2001 and 2002; Baye et al., 2003, Mathä, 2003; Gajewski and Kowalski, 2004; Isgut, 2004; Engel and Rogers, 2004; or Allington et al., 2005).

One market that has attracted particular interest is the European car market. A number of studies have focused on price convergence in this market (Gaulier and Haller, 2000; Lutz, 2003 and 2004; Goldberg and Verboven, 2001, 2004 and 2005). The automobile industry provides a good opportunity for studying price convergence within both EU and EMU countries. On the one hand, cross-country price differentials in the automobile industry are an important source of concern under the European Commission's competition policy. On the other hand, since May 1993, the European Commission publishes, twice a year, car price surveys for most car models sold in the EU. These surveys represent one of the rare comprehensive public sources of information on product prices in the EU at such detailed level. 
The aim of this paper is to examine whether the EU15 and the eurozone car markets have become more integrated, using as a metric the dispersion of prices net of taxes. Several studies of cross-country price behaviour in the European car market have used the European Commission surveys. Our study extends those works by using a different approach to examining this data set and by incorporating the most recent information. In particular, unlike previous studies we use the concept of $\sigma$-convergence to individual car models and our data set includes 13 post-EMU and 7 post-euro price surveys.

Moreover, since the European Monetary System (EMS) represented an intermediary step to the EMU, fostering economic integration and economic policy coordination in the EU, we devote particular attention to the convergence of prices experienced by countries whose currencies participated in the core of EMS. In this regard, it has been claimed that international trade in a regime of relatively fixed exchange rates such as that established by EMS would result in price convergence. Therefore, by analysing price dispersion among EMS countries with different degrees of exchange rate stability we hope to shed new light on the success of this exchange rate agreement in terms of imposing price discipline among its members.

The paper is organised as follows. Section 2 shortly discusses economic incentives and institutions generating deviations from the law of one price. Section 3 presents the data and Section 4 sets out the empirical findings. Finally, Section 5 offers some concluding remarks.

\section{The law of one price and the European car market}

Much work in international economics has focused on testing the validity of the law of one price across countries. There are two versions of the law of one price: the 
absolute and the relative versions. The absolute version states that, in the absence of transfer costs and under competitive conditions, identical tradable goods priced in a common currency should be equal across countries. The intuition is that international arbitrage should work until prices are aligned. In its relative form, the law of one price asserts that common currency prices for a particular product should change in the same way over time in different countries and, therefore, it is compatible with the existence of a stable price differential across markets.

Most of the empirical literature on the law of one price examines the validity of its relative version for two main reasons. First, arbitrage is not costless. Trading between locations itself has costs (such as transportation costs and trade barriers), so prices are very unlikely to be identical across locations. However, these costs may give rise to a stable price differential across markets. Second, the preference for testing the relative version is a consequence of data limitations rather than research interest. Typically, the data employed in price comparisons is in the form of price indices in different countries whose levels are arbitrary. However, in this paper, the price information is based on recommended retail prices of specific car models. The prices used are in ECUs/euros, net of taxes and have been adjusted for equipment differences. It allows us to focus on the analysis of the convergence to the absolute version of the law of one price.

Two conditions are necessary for the existence of international price differences beyond transfer costs. First, firms must have market power as well as some profit incentives to set different prices in different countries. Different demand elasticities, import quotas, or an incomplete pass-through (of taxes or exchange rates) are the most frequently studied sources of markup differences across countries in the European car market and, therefore, of international price discrimination. Second, firms must be able to prevent arbitrage. Traditionally, several non-tariff barriers, such as the type approval 
or the national registration, have contributed to keep European car markets geographically segmented. Despite the removal of a great part of the barriers within the EU in 1993, some obstacles to cross-border trade have remained since then. In particular, during the sample period the major obstacle to cross-border trade stems from the selective and exclusive distribution system authorised by Regulation 123/85 (from 1985 to 1995 ) and $1475 / 95$ (from 1995 up to 30 September 2002). This distribution system aimed to restrict sales of new cars in the EU to dealers chosen by manufacturers, becoming very difficult for independent wholesalers to buy cars in bulk in one country and resell them in another. An important liberalization of the distribution system was introduced in 2002 with transitions periods of at least one year. ${ }^{1}$

\section{Data}

The price data used in this study come from the biannual surveys of car price differentials between EU member States carried out by the European Commission since 1993. The methodology used has remained the same for all the surveys over the period 1993-2005. About 17 European and 8 Japanese manufacturers submit the recommended retail prices on 1 May and 1 November of each year of their top-selling products. The number of car models included in these surveys ranges from 72 to 91 . The prices are adjusted for equipment differences and are given in local currency and in ECU/euros, both before and after tax. It shall be noted that actual retail prices may differ from recommended list prices, as dealers are free to set their own prices. In particular, the data set on pre-tax list prices used in this study consists of the surveys conducted over the period from May 1995 to May 2005 (21 bi-annual surveys). The countries covered

\footnotetext{
${ }^{1}$ See Goldberg and Verboven (2004) for a detailed discussion about both the profit incentives for price discrimination in the car market and the European institutions that have made international price discrimination feasible in this industry.
} 
are all the EU-15 Member States. ${ }^{2}$ The car models considered are those with appropriate data in the range of countries and periods for the purposes of this paper. As a result, we have selected a sample of 45 models.

\section{Empirical results}

There are various ways of measuring price dispersion, for example, the range of minimum price to maximum price, the ratio of maximum price to minimum price, the ratio of maximum price to mean price, the standard deviation, or the coefficient of variation. We use the coefficient of variation (the ratio of the standard deviation to the mean) as a measure of price dispersion because it has advantages over the cited alternative measures. The coefficient of variation is invariable to changes of scale, which is useful for comparing price dispersion across products or, for a given product, price dispersion over time. This affords an advantage with respect to the range and the standard deviation. Moreover, although the range and max-min ratio provide a measure of the total spread of the data, they only take into account the two extreme values of the data, and, therefore, they are susceptible to considerable distortion if there is an unusual extreme observation. Similarly, the max-mean ratio only considers all the observations in the computation of the mean, in contrast to the coefficient of variation, which takes into account each of the data observations in both the numerator (which measures the average spread around the mean) and the denominator (the mean).

For the purpose of assessing price convergence in the European car market we use the concept of $\sigma$-convergence. ${ }^{3}$ Thus, to measure the degree of price convergence for

\footnotetext{
2 The pre-tax car prices are available since May 1993 for 10 European countries (Belgium, France, Germany, Ireland, Italy, Luxembourg, the Netherlands, Portugal, Spain, the United Kingdom). Pre-tax prices for Denmark and Greece are available since November 1994, while for Austria, Finland and Sweden they are available since May 1995. Data for the ten new members of the EU (Cyprus, Czech
} 
each car model, first, we calculate the coefficient of price variation for each bi-annual survey across the corresponding set of European markets. Then, the time series of coefficients of variation for each car model $\left(\mathrm{CV}_{\mathrm{t}}\right)$ are regressed on a constant and a linear time trend (TIME):

$$
C V_{t}=\alpha+\sigma T I M E+u_{t}
$$

where $\alpha$ and $\sigma$ are parameters to be estimated, and $\mathrm{u}_{\mathrm{t}}$ is the error term. If price dispersion declined steadily, we would expect the regression to yield a negative and statistically significant coefficient for the linear time trend.

Tables 1-4 present the results for the 45 car models considered in five different samples of countries. The first table presents the results for EU15. Table 2 focuses on EMU11 countries. Table 3 ("group A") examines price convergence for EU15 countries whose currencies participated continuously in the Exchange Rate Mechanism (ERM) of the EMS from the outset maintaining broadly stable bilateral exchange rates among themselves (Austria, Belgium, Denmark, France, Germany, Luxembourg, and the Netherlands). Finally, Table 4 ("group B") includes EU15 countries whose currencies showed considerable fluctuations in value relative to the German mark (Finland, Greece, Ireland, Italy, Portugal, Spain, Sweden, and the United Kingdom). ${ }^{4}$ Each table reports the estimated coefficients on the linear trend in the coefficients of variation for six periods. The first three periods (columns 1 to 3) start in 1995, ending in 1998 (the year before irrevocable exchange rates among EMU countries are fixed), 2001 (the year prior

Republic, Estonia, Latvia, Lithuania, Hungary, Malta, Poland, Slovakia, Slovenia) are not considered given that they are only available since May 2004.

${ }^{3} \sigma$-convergence occurs if the cross-sectional dispersion of a variable decreases over time.

${ }^{4}$ It is interesting to note that these two groups roughly correspond to the distinction made by the European Commission (1995) between those countries whose currencies continuously participated in the ERM from its inception maintaining broadly stable bilateral exchange rates among themselves over the sample period, and those countries whose currencies either entered the ERM later or suspended its participation in the ERM, as well as fluctuating in value to a great extent relative to the Deutschmark. These two groups are also basically the same found in Jacquemin and Sapir (1996), applying multivariate analysis 
to the introduction of the euro) and 2005 (the last year in the sample), respectively. In column 4 we analyse price convergence over the period 1998-2005. Finally, in columns 5 and 6 this recent period is divided into two which allows us to distinguish the first years of the EMU (when the euro did not circulated) and from that time onwards.

In general, the evidence of price dispersion trends varies both according to the sample of countries and the periods considered. As can be observed in Table 1, during the period 1995-1998 about only half of the estimates are negative and just 5 of them are statistically significant at the $10 \%$ level. Furthermore, 4 of the 21 positive coefficients reach the statistical significance. Therefore, it is not surprising that the average coefficient of variation in $1995(0.1008)$ be very similar to that found in $1998(0.1002)$. One fact that may help to explain the scarce evidence of convergence during this period is the presence of an incomplete pass-through of exchange rates to prices. Several studies for the automobile industry have found an incomplete degree of exchange rate pass-through to import prices (Gross and Schmitt, 1996; Gron and Swenson, 1996; Feenstra, et al., 1996), and the existence of international price discrimination induced by exchange rate movements (Gagnon and Knetter, 1995; Gil-Pareja, 2001; Gil-Pareja, 2003), a phenomenon termed pricing to market. ${ }^{5}$ Extending the period to 2001, we find an increase in the number of negative and statistically significant coefficients, but the distribution of positive and negative coefficients remains unchanged. However, when we add to the sample period data from 2002 onwards, the existence of price convergence is evident: 44 out of 45 coefficients are negative and $89 \%$ of them are

techniques (i.e., principal components and cluster analysis) to a wide set of structural and macroeconomic indicators, to form an homogeneous group of countries.

${ }^{5}$ In particular, Gil-Pareja (2003) investigates pricing to market behaviour in European car markets during the period 1993-1998 using also the bi-annual data provided by the European Commission surveys. He concludes that local currency price stability is a strong and pervasive phenomenon across products that is consequence, at least in part, of the existence of market segmentation and international price discrimination, despite the completion of the single market programme in 1993. 
statistically significant. A similar result emerges when we analyse price convergence over the period 1998-2005, for which the estimated coefficients for the linear time trend is negative in all the cases and they are statistically significant at the $1 \%$ level in $87 \%$ of them. Splitting this period into two we find a clear evidence of price convergence in both sub-periods although it is stronger after 2001 than from 1998 to 2001.

In comparison with the EU15, the sample of EMU11 countries (Table 2) shows a remarkably greater number of negative coefficients during the periods $1995-1998$ and 1995-2001 while for the full sample period the number of negative trends is slightly smaller. This result suggests that EMU countries started convergence previously to the EU as a whole, which, as noted before, does not show evidence of convergence before 1999. Moreover, the study of price convergence after that year provides broadly the same picture than for the EU in its entity. Nonetheless, two comments are in order. First, there is more evidence of convergence among the EU15 than among EMU countries. This might be expected since in 1998 price dispersion was considerably smaller across EMU countries (0.0669) than across EU member States (0.1002). Second, as was expected the evidence of price convergence is stronger in the post-euro period. ${ }^{6}$ The inclusion of Greece among the set of EMU countries leads to the same conclusions. ${ }^{7}$

Another important issue that is an aim of this paper is the analysis of the impact of exchange rates on convergence patterns. To this end, we study whether EU15 countries with relatively stable, credible exchange rates prior to the EMU (group A) showed a stronger tendency towards convergence than countries with relatively volatile

\footnotetext{
${ }^{6}$ Friberg (2001) provides theoretical support to the notion that the single currency should reduce the potential for price discrimination across participating countries. He shows that a monetary union promotes market integration by reducing the option value of segmenting markets.

${ }^{7}$ To economise on space, we do not report the results including Greece among the set of EMU countries, but they are available from the authors upon request.
} 
exchange rates (group B) ${ }^{8}$. In the stable currencies group (Table 3), a small majority, 26 out of 45, cases show a declining trend over the period 1995-1998, although only 10 of them are statistically significant at the $10 \%$ level. However, in group B (Table 4) only 6 estimates are negative and only one of them is statistically significant at conventional levels. In contrast, in the last group 24 out of 39 positive coefficients reach the statistical significance at the $10 \%$ level. ${ }^{9}$ The enlargement of the sample period to 2001 increases the number of significant trends towards lower price dispersion in both groups, even though it is worth noting that there is again more evidence of convergence in group A (with 19 negative and statistically significant estimates) and that, in group B, 16 of the 34 positive coefficients are statistically significant. When we extend the sample period to 2005 , we observe significantly lower price dispersion over time in 35 (23) car models in group A (B). Focussing attention on the period 1998-2005, the results indicate a significant trend toward lower dispersion in both samples, but in this case it is particularly intense in group B where it occurs in 42 models. As a result of these trends the average coefficient of variation in 2005 in group B (0.0561) is slightly smaller than in group A (0.0577). Finally, it is worth noting that the evidence that emerges after splitting this period into two does not differ markedly in both groups of countries, being greater the number of negative trends in the post-euro period.

\section{Concluding remarks}

This paper has offered empirical evidence of price convergence in one market that has attracted special attention during the last decade: the European car market. In particular, we have examined whether the EU15 and the eurozone car markets have

\footnotetext{
${ }^{8}$ See Ledesma-Rodríguez et al. (2005) for an empirical evaluation of the credibility of the commitment to maintain the exchange rate around a central parity in the ERM.
} 
become more integrated, using a different approach to that employed in previous works (the concept of $\sigma$-convergence to individual car models) and incorporating the most recent information. We have also explored the effect of exchange rates on price convergence patterns by reviewing the experience of European countries that participated in the exchange rate stability zone with a high degree of confidence with respect to the commitment to maintain the exchange rate around a central parity against countries with relatively volatile exchange rates.

Overall, the evidence of price convergence varies both according to the sample of countries and the periods considered. In the EU as a whole there is no tendency for price dispersion to fall over the period 1995-1998. In contrast, from 1998 onwards the evidence of price convergence is pervasive and stronger in the post euro period than until 2001.

If we restrict the sample to the EMU markets, we observe significant lower price dispersion over time since 1995. It suggests that EMU countries started convergence previously to the EU as a whole. However, the study of price convergence after irrevocable exchange rates were fixed provides broadly the same picture than for the EU in its entity, being the average coefficient of variation in 2005 slightly smaller in EMU countries.

Finally, exchange rate movements over the period 1995-1998 has significantly contributed to price dispersion across countries. In particular, countries with relatively volatile exchange rates show a tendency towards a higher price dispersion over the period 1995-1998. However, from 1998 onwards there is a very clear evidence of lower price dispersion over time.

\footnotetext{
${ }^{9}$ Consistent with these trends, the average coefficient of variation in group A fall from 0.095 to 0.087 over the period 1995-1998, whereas, in group B, it increases from 0.079 to 0.108 over the same period.
} 


\section{References}

Allington, N. F. B., Kattuman, P. A. and Waldmann, F. A. (2005): “One market, one money, one price? Price dispersion in the European Union", The Judge Institute of Management, University of Cambridge, Working Paper P 01/2005.

Baye, M., Gatti, R., Kattuman, P. and Morgan, J. (2003): "Did the euro foster online price competition? Evidence from an International Price Comparison Site", forthcoming in Economic Inquiry.

Camarero, M., Esteve, V. and Tamarit, C. (2000): "Price convergence of the peripheral European countries on the way to the EMU: A time series approach", Empirical Economics 25, pp. 149-168.

Chen, N. (2004): “The behaviour of relative prices in the European Union: A sectoral analysis", European Economic Review 48, pp. 1257-1286.

Engel, C. and Rogers, J. H. (2004): "European product market integration after the euro", Economic Policy 19, pp. 347-384.

European Commission (1995): "The impact of exchange rate movements on trade within the single market", European Economy, Reports and Studies, No. 4.

Feenstra, R. C., Gagnon, J. E. and Knetter, M. M. (1996): "Market share and exchange rate pass-through in world automobile trade", Journal of International Economics 40, pp. 187-207.

Friberg, R. (2001): "Two monies, two markets? Variability and the option to segment", Journal of International Economics 55, pp. 317-327.

Gagnon, J. E. and Knetter, M. M. (1995): "Markup adjustment and exchange rate fluctuations: evidence from panel data on automobile exports", Journal of International Money and Finance 14, pp. 289-310. 
Gámez-Amián, C. and Morales-Zumaquero, A. (2002): Complete or partial inflation convergence in the EU?, Working paper E2002/09, Sevilla, CentrA.

Gajewski, P. and Kowalski, J. (2004): Price convergence in the European Union countries, Institute of Economics, University of Lodz, mimeo.

Gaullier, G. and Haller, S. (2000): "The convergence of automobile prices in the European Union: an empirical analysis for the period 1993-1999”, CEPII, Working Paper 2000-14.

Gil-Pareja, S. (2001): "Pricing to market in European automobile exports to OECD countries: a panel data approach", Applied Economics 14, pp. 1845-1856.

Gil-Pareja, S. (2003): "Pricing to market behaviour in European car markets", European Economic Review 47, pp. 945-962.

Gil-Pareja, S. and Sosvilla-Rivero, S. (2004): "Export market integration in the European Union”, Journal of Applied Economics 7, pp. 271-301.

Goldberg, P. K. and Verboven, F. (2001): "The evolution of price dispersion in the European car market", Review of Economic Studies 68, pp. 811-848.

Goldberg, P. K. and Verboven, F. (2004): "Cross-country price dispersion in the euro era: a case study of the European car market", Economic Policy 19, pp. 483-521.

Goldberg, P. K. and Verboven, F. (2005): "Market integration and convergence to the law of one price: evidence from the European car market", Journal of International Economics 65, pp. 49-74 .

Gron, A. and Swenson, D. L. (1996): "Incomplete exchange rate pass-through and imperfect competition: the effect of local production", American Economic Review 86, pp. 71-76.

Gross, D. G. and Schmitt, N. (1996): "Exchange rate pass-through and rivalry in the Swiss automobile market”, Weltwirtschaftliches Archiv 132, pp. 278-303. 
Isgut, I. (2004): “Common currencies and market integration across cities: How strong is the link?", in V. Alexander, J. Mélitz, and G. M. von Furstenberg (eds.), Monetary Unions and Hard Pegs: Effects on Trade, Financial Development, and Stability (Oxford: Oxford University Press), Chapter 8.

Jacquemin, A. and Sapir, A. (1996): "Is a European hard core credible? A statistical analysis", Kyklos 49, pp. 105-117.

Ledesma-Rodríguez, F., Navarro-Ibáñez, M., Pérez-Rodríguez, J., and Sosvilla-Rivero, S. (2005): "Assessing the Credibility of a Target Zone: Evidence from the EMS", forthcoming in Applied Economics.

Lutz, M. (2003): Price convergence under the EMU? First estimates, Discussion Paper No. 2003-08, Department of Economics, University of St. Gallen, Switzerland.

Lutz, M. (2004): "Pricing in segmented markets, arbitrage barriers and the law of one price: evidence from the European car market", Review of International Economics 12 pp. $456-475$.

Mathä, T. Y. (2003): What of expect to the euro? Analysing price differences of individual products in Luxembourg and its surrounding regions, Banque Centrale du Luxembourg, Working Paper 8.

Parsley, D. C. and Wei, S. J. (2001): Limiting currency volatility to stimulate goods market integration, NBER Working Paper No. 8468.

Rogers, J. H. (2001): "Price level convergence, relative prices, and inflation in Europe", International Finance Discussion Paper 699, Board of Governors of the Federal Reserve System.

Rogers, J. H. (2002): Monetary union, price level convergence, and inflation: How close is Europe to the United States", Board of Governors of the Federal Reserve System, International Finance discussion Paper No. 740. 
Rogers, J. H., Hufbauer, G. C. and Wada, E. (2001): Price level convergence and inflation in Europe, Working Paper 01-1, Institute for International Economics.

Sosvilla-Rivero, S. and Gil-Pareja, S. (2004): "Price convergence in the European Union”, Applied Economics Letters 11, pp. 39-47. 
Table 1. Trends in price dispersion. Sample: EU15.

\begin{tabular}{|c|c|c|c|c|c|c|}
\hline Model & (1) & (2) & (3) & (4) & (5) & (6) \\
\hline & 1995-1998 & 1995-2001 & $1995-2005$ & $1998-2005$ & 1998-2001 & $2001-2005$ \\
\hline Alfa 145/147 & $0.0099 * *$ & 0.0030 & -0.0018 & $-0.0063 * * *$ & $-0.0068 * *$ & $-0.0037 * * *$ \\
\hline Alfa $155 / 156$ & 0.0043 & $0.0020 * *$ & $-0.0029 * * *$ & $-0.0059 * * *$ & -0.0011 & -0.0058 \\
\hline Audi A4 & $-0.0026 * *$ & $-0.0036 * * *$ & $-0.0042 * * *$ & $-0.0045 * * *$ & $-0.0035 * *$ & $-0.0051 * * *$ \\
\hline Audi A6 & $0.0031 *$ & $-0.0017 *$ & $-0.0026 * * *$ & $-0.0039 * * *$ & $-0.0043 *$ & $-00064 * * *$ \\
\hline BMW 3-series & -0.0013 & $-0.0014 *$ & $-0.0024 * * *$ & $-0.0028 * * *$ & -0.0008 & $-0.0037 * * *$ \\
\hline BMW 5-series & -0.005 & $-0.0024 *$ & $-0.0031 * * *$ & $-0.0034 * * *$ & $-0.0025^{*}$ & $-0.0043 * * *$ \\
\hline BMW 7-series & -0.0013 & $-0.0056^{*}$ & $-0.0043 * * *$ & $-0.0031 * * *$ & $-0.0058 * * *$ & -0.00012 \\
\hline Citroën AX/saxo/C2 & -0.0026 & -0.0004 & $-0.0025 * * *$ & $-0.0044 * * *$ & -0.0014 & $-0.0060^{*}$ \\
\hline Citroën ZX/ Xsara/C4 & -0.0028 & 0.0020 & -0.0015 & $-0.0044 * * *$ & 0.0017 & $-0.0090 * * *$ \\
\hline Citroën Xantia/C5 & $-0.0060 * * *$ & 0.0012 & $-0.0014 *$ & $-0.0030 * *$ & 0.0032 & $-0.0054 * * *$ \\
\hline Fiat Cinq./Seicento/Panda & -0.0036 & $0.0038^{* *}$ & -0.0001 & -0.0011 & $0.0123 * * *$ & $-0.0092 * * *$ \\
\hline Fiat Punto & 0.0033 & 0.0021 & $-0.0018 *$ & $-0.0047 * * *$ & -0.0021 & -0.0018 \\
\hline Fiat Bravo/Stilo & $0.0078 * *$ & 0.0030 & -0.0016 & $-0.0065 * * *$ & -0.0057 & -0.0050 \\
\hline Ford Fiesta & 0.0043 & -0.0018 & $-0.0029 * * *$ & $-0.0037 * * *$ & $-0.0046 * *$ & 0.0000 \\
\hline Ford Escort/Focus & 0.0019 & -0.0007 & $-0.0021 * * *$ & $-0.0029 * * *$ & -0.0016 & $-0.0032 * * *$ \\
\hline Ford Mondeo & 0.0030 & -0.0016 & $-0.0033 * * *$ & $-0.0052 * * *$ & $-0.0055 * * *$ & $-0.0059 * * *$ \\
\hline Honda Civic & 0.0040 & -0.0017 & $-0.0030 * * *$ & $-0.0054 * * *$ & $-0.0097 * * *$ & -0.0018 \\
\hline Honda Accord & -0.0017 & 0.0008 & $-0.0019 * * *$ & $-0.0038 * * *$ & 0.0005 & $-0.0067 * * *$ \\
\hline Mazda 3-series & $0.0061 * * *$ & 0.0027 & $-0.0029 * *$ & $-0.0079 * * *$ & -0.0035 & $-0.0076 * * *$ \\
\hline Mercedes 180/c180 & $-0.0039 *$ & $-0.0024 * * *$ & $-0.0016 * * *$ & $-0.0010 * * *$ & $-0.0018^{*}$ & -0.0004 \\
\hline Mercedes 200E/E220 & $-0.0055^{* *}$ & $-0.0032 * * *$ & $-0.0027 * * *$ & $-0.0017 * * *$ & -0.0004 & $-0.0017 *$ \\
\hline Mercedes 320S/S350 & -0.0021 & $-0.0038^{* * *}$ & $-0.0031 * * *$ & $-0.0329 * * *$ & $-0.0052 * *$ & $-0.0011 *$ \\
\hline Nissan Micra & -0.0018 & -0.0012 & $-0.0019 * * *$ & -0.0014 & $0.0027^{*}$ & $-0.0039 * *$ \\
\hline Nissan Sunny/Almera & 0.0028 & $0.0021 *$ & $-0.0014 *$ & $-0.0034 * * *$ & 0.0026 & $-0.0064 * * *$ \\
\hline Nissan Primera & -0.0007 & 0.0012 & $-0.0014 * *$ & $-0.0024 * *$ & $0.0044 *$ & $-0.0053 * * *$ \\
\hline Opel Corsa & -0.0010 & -0.0004 & $-0.0034 * * *$ & $-0.0047 * * *$ & 0.0015 & $-0.0067 * * *$ \\
\hline Opel Astra & 0.0022 & 0.0009 & $-0.0018 * * *$ & $-0.0038 * * *$ & -0.0012 & $-0.0041 *$ \\
\hline Opel Vectra & 0.0017 & 0.0005 & $-0.0023 * * *$ & $-0.0038 * * *$ & 0.0012 & $-0.0061^{* *}$ \\
\hline Opel Omega/Signum & -0.0008 & 0.0006 & $-0.0022 * * *$ & $-0.0042 * * *$ & 0.0005 & $-0.0072 * * *$ \\
\hline Peugeot 306/307 & 0.0030 & $0.0034 * * *$ & -0.0007 & $-0.0039 * * *$ & 0.0021 & $-0.0076 * * *$ \\
\hline Peugeot $405 / 406 / 407$ & $-0.0043^{*}$ & $0.0033 * * *$ & 0.0012 & -0.0002 & $0.0072 * * *$ & $-0.0097 *$ \\
\hline Renault Clio & 0.0020 & $0.0016^{*}$ & $-0.0014 * *$ & $-0.0037 * * *$ & -0.0002 & $-0.0039 * *$ \\
\hline Renault 19/Megane & 0.0030 & 0.0008 & $-0.0020 * * *$ & $-0.0039 * * *$ & 0.0006 & $-0.0062^{*}$ \\
\hline Renault Laguna & -0.0006 & 0.0021 & $-0.0018 * *$ & $-0.0043 * * *$ & 0.0027 & $-0.0056 * * *$ \\
\hline Range Rover & -0.0065 & -0.0043 & $-0.0077 * * *$ & $-0.0139 * * *$ & -0.0224 & $-0.0039 * * *$ \\
\hline Seat Ibiza & -0.0014 & $-0.0026^{*}$ & $-0.0034 * * *$ & $-0.0048 * * *$ & $-0.0062 *$ & -0.0017 \\
\hline Seat Cordoba & 0.0012 & -0.0014 & $-0.0034 * * *$ & $-0.0053 * * *$ & $-0.0057^{*}$ & -0.0022 \\
\hline Seat Toledo & -0.0015 & -0.0009 & $-0.0027 * * *$ & $-0.0040 * * *$ & -0.0017 & $-0.0030 * * *$ \\
\hline Toyota Starlet/Yaris & -0.0005 & $-0.0034 *$ & $-0.0039 * * *$ & -0.0040 *** & $-0.0037 *$ & $-0.0028 * * *$ \\
\hline Toyota Corola & -0.0007 & -0.0012 & $-0.0026 * * *$ & $-0.0041 * * *$ & -0.0033 & -0.0037 \\
\hline Toyota Carina/Avensis & 0.0007 & 0.0006 & $-0.0023 * * *$ & $-0.0049 * * *$ & -0.0027 & $-0.0057 * * *$ \\
\hline Volvo 440/s40 & -0.0048 & $-0.0032 * * *$ & $-0.0017 * *$ & -0.0008 & -0.0022 & -0.0016 \\
\hline VW Polo & 0.0024 & $-0.0038 * * *$ & $-0.0037 * * *$ & $-0.0046 * * *$ & $-0.0103 * * *$ & 0.0011 \\
\hline VW Golf & $0.0067 * * *$ & 0.0006 & $-0.0014 * *$ & $-0.0040 * * *$ & $-0.0062 * * *$ & $-0.0014 * * *$ \\
\hline VW Passat & 0.0045 & -0.0017 & $-0.0021 * * *$ & $-0.0031 * * *$ & $-0.0066 * * *$ & -0.0002 \\
\hline
\end{tabular}

Notes: * significant at 10\%; ** significant at 5\%; *** significant at $1 \%$. Each trend is the coefficient estimate of biannual price dispersion (measured as the coefficient of variation) regressed on a time trend. The regressions include a constant. 
Table 2. Trends in price dispersion. Sample: EMU11

\begin{tabular}{|c|c|c|c|c|c|c|}
\hline Model & (1) & (2) & (3) & (4) & (5) & (6) \\
\hline & 1995-1998 & $1995-2001$ & $1995-2005$ & 1998-2005 & $1998-2001$ & $2001-2005$ \\
\hline Alfa 145/147 & -0.0011 & -0.0003 & $-0.0013 *$ & $-0.0033 * * *$ & $-0.0062 *$ & -0.0009 \\
\hline Alfa $155 / 156$ & $-0.0059 * * *$ & -0.0008 & -0.0010 & -0.0002 & $0.0024 *$ & 0.0004 \\
\hline Audi A4 & $-0.0020^{* *}$ & $-0.0056^{* * * *}$ & $-0.0040 * * *$ & $-0.0031 * * *$ & $-0.0070 * *$ & -0.0006 \\
\hline Audi A6 & 0.0025 & $-0.0035 * * *$ & $-0.0028 * * *$ & $-0.0026 * *$ & -0.0050 & $-0.0042 * * *$ \\
\hline BMW 3-series & -0.0008 & -0.0009 & $-0.0011 * * *$ & $-0.0014 * * *$ & $-0.0014 * *$ & $-0.0015^{*}$ \\
\hline BMW 5-series & -0.0022 & $-0.0015^{*}$ & $-0.0019 * * *$ & $-0.0025 * * *$ & $-0.0019 * * *$ & $-0.0032 * * *$ \\
\hline BMW 7-series & -0.0019 & $-0.0043 * * *$ & $-0.0042 * * *$ & $-0.0047 * * *$ & $-0.0073 * * *$ & -0.0024 \\
\hline Citroën $\mathrm{AX} / \mathrm{saxo} / \mathrm{C} 2$ & $-0.0076 * * *$ & $-0.0063 * * *$ & $-0.0033 * * *$ & $-0.0016^{*}$ & $-0.0066 * * *$ & 0.0009 \\
\hline Citroën ZX/ Xsara/C4 & $-0.0067 * * *$ & $-0.0026 * * *$ & $-0.0025 * * *$ & $-0.0024 * * *$ & -0.0010 & $-0.0029 * * *$ \\
\hline Citroën Xantia/C5 & $-0.0062 * * *$ & $-0.0020 * *$ & $-0.0031 * * *$ & $-0.0036 * * *$ & -0.0002 & $-0.0048 * * *$ \\
\hline Fiat Cinq./Seicento/Panda & $-0.0082 * * *$ & -0.0000 & -0.0004 & 0.0005 & $0.0071 * * *$ & $-0.0052 * * *$ \\
\hline Fiat Punto & $-0.0081 * * *$ & $-0.0032 * * *$ & $-0.0014 * * *$ & 0.0004 & 0.0007 & 0.0003 \\
\hline Fiat Bravo/Stilo & -0.0013 & -0.0016 & -0.0006 & 0.0002 & -0.0017 & 0.0006 \\
\hline Ford Fiesta & 0.0015 & $-0.0049 * * *$ & $-0.0034 * * *$ & $-0.0021 * *$ & $-0.0061 * * *$ & 0.0009 \\
\hline Ford Escort/Focus & $-0.0036^{*}$ & $-0.0027 * * *$ & -0.0007 & 0.0010 & -0.0006 & -0.0002 \\
\hline Ford Mondeo & -0.0017 & $-0.0033 * *$ & $-0.0026 * * *$ & $-0.0020 * * *$ & -0.0029 & $-0.0029 * * *$ \\
\hline Honda Civic & -0.0017 & $-0.0024 * * *$ & $-0.0016 * * *$ & $-0.0018 * * *$ & $-0.0044 * * *$ & -0.0006 \\
\hline Honda Accord & -0.0011 & 0.0000 & -0.0006 & -0.0009 & 0.0019 & $-0.0055^{* * * *}$ \\
\hline Mazda 3-series & 0.0031 & 0.0014 & -0.00011 & $-0.0032 * * *$ & -0.0005 & $-0.0038 * *$ \\
\hline Mercedes 180/c180 & $-0.0056 * * *$ & $-0.0027 * * *$ & $-0.0018 * * *$ & $-0.0011 * * *$ & -0.0014 & $-0.0009 * * *$ \\
\hline Mercedes 200E/E220 & $-0.0051 * *$ & $-0.0042 * * *$ & $-0.0029 * * *$ & $-0.0017 * * *$ & $-0.0026 * * *$ & $-0.0008 * * *$ \\
\hline Mercedes $320 \mathrm{~S} / \mathrm{S} 350$ & -0.0019 & $-0.0048 * * *$ & $-0.0035 * * *$ & $-0.0031 * * *$ & $-0.0072 * * *$ & $-0.0005 * * *$ \\
\hline Nissan Micra & -0.0033 & $-0.0033 * * *$ & $-0.0012 * * *$ & 0.0003 & $-0.0027 * * *$ & 0.0016 \\
\hline Nissan Sunny/Almera & -0.0005 & 0.0003 & 0.0001 & 0.0004 & $0.0022 *$ & -0.0002 \\
\hline Nissan Primera & $-0.0045 * *$ & -0.0002 & -0.0000 & 0.0007 & $0.0032 * * *$ & -0.0002 \\
\hline Opel Corsa & $-0.0048 * *$ & -0.0005 & $-0.0020 * * *$ & -0.0016 & $0.0055^{*}$ & $-0.0059^{* *}$ \\
\hline Opel Astra & -0.0012 & 0.0004 & $-0.0013^{* *}$ & $-0.0022 * *$ & 0.0028 & $-0.0075 * * *$ \\
\hline Opel Vectra & -0.0009 & 0.0000 & $-0.0017 * * *$ & $-0.0019 *$ & $0.0041^{*}$ & $-0.0062 * * *$ \\
\hline Opel Omega/Signum & $-0.0031 * *$ & -0.0002 & $-0.0013 * *$ & -0.0016 & 0.0031 & $-0.0075 * * *$ \\
\hline Peugeot 306/307 & $-0.0037 *$ & $-0.0017 * *$ & $-0.0013 * * *$ & $-0.0007 *$ & 0.0006 & $-0.0018 * * *$ \\
\hline Peugeot 405/406/407 & $-0.0041 *$ & -0.0006 & -0.0000 & 0.0004 & 0.0022 & -0.0061 \\
\hline Renault Clio & -0.0030 & 0.0002 & -0.0005 & -0.0003 & $0.0034 * * *$ & -0.0020 \\
\hline Renault 19/Megane & $\begin{array}{ll}-0.0038 \\
\end{array}$ & -0.0003 & -0.0006 & -0.0004 & $0.0024 * * *$ & $-0.0029 * * *$ \\
\hline Renault Laguna & $-0.0079 * * *$ & $-0.0025 *$ & $-0.0019 * * *$ & -0.008 & 0.0022 & -0.0012 \\
\hline Range Rover & -0.0107 & -0.0085 & $-0.0088^{* * *}$ & $-0.0126 * * *$ & -0.0246 & $-0.0032 * *$ \\
\hline Seat Ibiza & -0.0025 & -0.0007 & $-0.0012 * *$ & $-0.0021 * * *$ & -0.0022 & $-0.0013 *$ \\
\hline Seat Cordoba & -0.0039 & -0.0005 & -0.0007 & -0.0007 & 0.0006 & 0.0002 \\
\hline Seat Toledo & -0.0058 **** & $-0.0018 * *$ & $-0.0018 * * *$ & $-0.0013 * * *$ & 0.0009 & -0.0017 \\
\hline Toyota Starlet/Yaris & 0.0021 & -0.0030 & $-0.0020^{*}$ & $-0.0009 * *$ & $-0.0028^{*}$ & -0.0001 \\
\hline Toyota Corola & 0.0008 & -0.0006 & 0.0002 & 0.0004 & $-0.0017 * *$ & 0.0000 \\
\hline Toyota Carina/Avensis & -0.0007 & 0.0005 & -0.0002 & $-0.0008 *$ & 0.0007 & $-0.0021 * * *$ \\
\hline Volvo 440/s40 & -0.0028 & $-0.0022 * *$ & 0.0001 & 0.0017 & -0.0010 & 0.0011 \\
\hline VW Polo & $-0.0039 * *$ & $-0.0049 * * *$ & $-0.0038 * * *$ & $-0.0031 * * *$ & $-0.0053 * * *$ & $-0.0018 *$ \\
\hline VW Golf & -0.0014 & $-0.0017 * * *$ & $-0.0014 * * *$ & $-0.0014 * * *$ & $-0.0022 *$ & $-0.0014 * * *$ \\
\hline VW Passat & -0.0013 & $-0.0038 * * *$ & $-0.0028 * * *$ & $-0.0017 * * *$ & $-0.0031 * *$ & -0.0011 \\
\hline
\end{tabular}

Notes: * significant at $10 \%$; ** significant at 5\%; *** significant at $1 \%$. Each trend is the coefficient estimate of biannual price dispersion (measured as the coefficient of variation) regressed on a time trend. The regressions include a constant. 
Table 3. Trends in price dispersion. Sample: group A

\begin{tabular}{|c|c|c|c|c|c|c|}
\hline Model & (1) & (2) & (3) & (4) & (5) & (6) \\
\hline & 1995-1998 & $1995-2001$ & $1995-2005$ & $1998-2005$ & $1998-2001$ & $2001-2005$ \\
\hline Alfa 145/147 & $0.0080 *$ & 0.0030 & -0.0006 & $-0.0049 * * *$ & $-0.0062 * *$ & $-0.0041 * * *$ \\
\hline Alfa $155 / 156$ & 0.0030 & 0.0011 & $-0.0033 * * *$ & $-0.0057 * * *$ & -0.0002 & -0.0066 \\
\hline Audi A4 & $-0.0031 * * *$ & $-0.0039 * * *$ & $-0.0045 * * *$ & $-0.0051 * * *$ & $-0.0054 * *$ & $-0.0036 * *$ \\
\hline Audi A6 & 0.0002 & $-0.0011 * *$ & $-0.0026 * * *$ & $-0.0039 * * *$ & -0.0014 & $-0.0077 * * *$ \\
\hline BMW 3-series & -0.0020 & $-0.0028 * * *$ & $-0.0030 * * *$ & $-0.0027 * * *$ & $-0.0015 * * *$ & $-0.0039 * * *$ \\
\hline BMW 5-series & -0.0013 & $-0.0020 * * *$ & $-0.0031 * * *$ & $-0.0036 * * *$ & $-0.0016^{*}$ & $-0.0056 * * *$ \\
\hline BMW 7-series & 0.0009 & -0.0002 & $-0.0011 * * *$ & $-0.0017 * * *$ & 0.0005 & $-0.0027 *$ \\
\hline Citroën AX/saxo/C2 & -0.0003 & 0.0015 & $-0.0016 * * *$ & $-0.0039 * * *$ & 0.0009 & $-0.0066 * * *$ \\
\hline Citroën ZX/ Xsara/C4 & 0.0013 & 0.0068 & 0.0004 & $-0.0039 *$ & $0.0091 * * *$ & $-0.0135 * * *$ \\
\hline Citroën Xantia/C5 & 0.0006 & $0.0070 * * *$ & 0.0014 & -0.0022 & 0.0076 & $-0.0036 * *$ \\
\hline Fiat Cinq./Seicento/Panda & $-0.0095 * * *$ & -0.0014 & $-0.0015 *$ & 0.0002 & 0.0067 & -0.0004 \\
\hline Fiat Punto & 0.0059 & 0.0001 & $-0.0018^{*}$ & $-0.0029 * *$ & -0.0021 & -0.0008 \\
\hline Fiat Bravo/Stilo & $-0.0032 * *$ & -0.0007 & -0.0010 & -0.0016 & -0.0017 & -0.0023 \\
\hline Ford Fiesta & 0.0003 & $-0.0024 * * *$ & $-0.0021 * * *$ & $-0.0021 * * *$ & $-0.0046 * * *$ & 0.0016 \\
\hline Ford Escort/Focus & $\begin{array}{ll}-0.0043 \\
\end{array}$ & $-0.0041 * * *$ & $-0.0029 * * *$ & $-0.0017 *$ & $\begin{array}{l}-0.0019 \\
\end{array}$ & $-0.0042 *$ \\
\hline Ford Mondeo & -0.0025 & $\begin{array}{ll}-0.0018 \\
\end{array}$ & $-0.0033 * * *$ & $-0.0043 * * *$ & -0.0007 & $-0.0090 * * *$ \\
\hline Honda Civic & 0.0008 & $-0.0048 * *$ & $-0.0028 * * *$ & -0.0019 & -0.0080 & -0.0008 \\
\hline Honda Accord & $-0.0083 * * *$ & -0.0030 & $-0.0024 * * *$ & -0.0015 & 0.0001 & $-0.0055 * * *$ \\
\hline Mazda 3-series & 0.0002 & -0.00002 & $-0.0022 * * *$ & $-0.0045 * * *$ & -0.0037 & -0.0036 \\
\hline Mercedes 180/c180 & $-0.0024 * * *$ & $-0.0024 * * *$ & $-0.0019 * * *$ & $-0.0017 * * *$ & $-0.0031 * * *$ & $-0.0007 * * *$ \\
\hline Mercedes 200E/E220 & -0.0010 & $-0.0017 * *$ & $-0.0014 * * *$ & $-0.0008 * * *$ & -0.0003 & $-0.0015 * * *$ \\
\hline Mercedes 320S/S350 & -0.0003 & $-0.0020 * * *$ & $-0.0012 * * *$ & -0.0005 & -0.0018 & 0.0001 \\
\hline Nissan Micra & $-0.0059 *$ & $-0.0047 * * *$ & $-0.0023 * * *$ & $\begin{array}{l}-0.0001 \\
\end{array}$ & -0.0020 & 0.0012 \\
\hline Nissan Sunny/Almera & -0.0062 & -0.0021 & $-0.0016 * * *$ & -0.0002 & 0.0032 & -0.0019 \\
\hline Nissan Primera & -0.0069 & 0.0009 & -0.0012 & -0.0010 & $0.0093 * * *$ & $-0.0067 * * *$ \\
\hline Opel Corsa & -0.0036 & -0.0008 & $-0.0027 * * *$ & $-0.0033 * * *$ & 0.0026 & $-0.0066 * * *$ \\
\hline Opel Astra & 0.0002 & 0.0001 & $-0.0012 *$ & $-0.0026 * * *$ & -0.0016 & -0.0033 \\
\hline Opel Vectra & 0.0009 & 0.0014 & $0.0025 * * *$ & $-0.0048 * * *$ & 0.0018 & -0.0061 \\
\hline Opel Omega/Signum & -0.0006 & -0.0007 & $-0.0026 * * *$ & $-0.0040 * * *$ & -0.0015 & $-0.0047 * * *$ \\
\hline Peugeot 306/307 & $0.0045 * * *$ & $0.0051 * * *$ & $0.0015^{*}$ & -0.0012 & 0.0045 & $-0.0065 * * *$ \\
\hline Peugeot 405/406/407 & 0.0046 & $0.0077 * *$ & $0.0045 * * *$ & 0.0009 & 0.0059 & -0.0074 \\
\hline Renault Clio & 0.0006 & -0.0003 & $-0.0029 * * *$ & $-0.0049 * * *$ & -0.0010 & $-0.0079 * * *$ \\
\hline Renault 19/Megane & 0.0030 & 0.0004 & $-0.0023 * *$ & $-0.0044 * * *$ & -0.0005 & $-0.0080^{*}$ \\
\hline Renault Laguna & 0.0009 & $0.0014 *$ & $-0.0016 * * *$ & $-0.0039 * * *$ & 0.0004 & $-0.0056 * * *$ \\
\hline Range Rover & $-0.0075^{* *}$ & 0.0017 & -0.0024 & $\begin{array}{l}-0.0048 \\
\end{array}$ & 0.0042 & $-0.0032 *$ \\
\hline Seat Ibiza & $-0.0058 * * *$ & $-0.0026^{* *}$ & $-0.0044 * * *$ & $-0.0053 * * *$ & -0.0020 & -0.0040 \\
\hline Seat Cordoba & $\begin{array}{l}-0.0010 \\
\end{array}$ & $\begin{array}{l}-0.0016 \\
-16\end{array}$ & $-0.0044 * * *$ & $-0.0064 * * *$ & -0.0034 & $-0.0062 * * *$ \\
\hline Seat Toledo & $-0.0054 * *$ & $-0.0024 *$ & $-0.0028 * * *$ & $-0.0024 *$ & 0.0015 & $-0.0046 * * *$ \\
\hline Toyota Starlet/Yaris & -0.0052 & $-0.0028 * *$ & $-0.0023 * * *$ & $-0.0015^{* *}$ & -0.0002 & $-0.0034 * *$ \\
\hline Toyota Corola & $-0.0072 * * *$ & $-0.0047 * * *$ & $-0.0034 * * *$ & -0.0023 & -0.0013 & -0.0061 \\
\hline Toyota Carina/Avensis & -0.0019 & $-0.0017 * * *$ & $-0.0031 * * *$ & $-0.0043 * * *$ & $-0.0019 * * *$ & $-0.0071 * * *$ \\
\hline Volvo $440 / \mathrm{s} 40$ & -0.0077 & $-0.0045^{* * * *}$ & $-0.0035 * * *$ & -0.0021 & 0.0005 & $-0.0068^{* *}$ \\
\hline VW Polo & -0.0017 & $-0.0014 * * *$ & $-0.0016^{* * * *}$ & $-0.0017 * *$ & $-0.0020^{*}$ & -0.0005 \\
\hline VW Golf & 0.0027 & $0.0016^{*}$ & $0.0007 *$ & $-0.0004 *$ & -0.0007 & -0.0005 \\
\hline VW Passat & 0.0007 & $-0.0014 * *$ & $-0.0011 * * *$ & $-0.0010 * * *$ & $-0.0024 * *$ & -0.0002 \\
\hline
\end{tabular}

Notes: * significant at 10\%; ** significant at 5\%; *** significant at 1\%. Each trend is the coefficient estimate of biannual price dispersion (measured as the coefficient of variation) regressed on a time trend. The regressions include a constant. Group A includes: Austria, Belgium, Denmark, France, Germany, Luxembourg, and the Netherlands. 
Table 4. Trends in price dispersion. Sample: group B

\begin{tabular}{|c|c|c|c|c|c|c|}
\hline Model & (1) & (2) & (3) & (4) & (5) & (6) \\
\hline & 1995-1998 & $1995-2001$ & $1995-2005$ & $1998-2005$ & $1998-2001$ & $2001-2005$ \\
\hline Alfa 145/147 & $0.0142 *$ & 0.0049 & -0.0023 & $-0.0089 * * *$ & -0.0090 & $-0.0041 * *$ \\
\hline Alfa $155 / 156$ & $0.0128 * *$ & $0.0057 * * *$ & -0.0011 & $-0.0059 * * *$ & -0.0016 & -0.0051 \\
\hline Audi A4 & 0.0010 & $-0.0023 *$ & $-0.0035 * * *$ & $-0.0041 * * *$ & -0.0021 & $-0.0069 * * *$ \\
\hline Audi A6 & $0.0104 * *$ & -0.0014 & $-0.0024 * *$ & $-0.0043 * * *$ & $-0.0080 * *$ & $-0.0053 * *$ \\
\hline BMW 3-series & -0.0005 & 0.0004 & $-0.0015 * *$ & $-0.0028 * * *$ & 0.0002 & $-0.0038 * * *$ \\
\hline BMW 5-series & 0.0009 & -0.0018 & $-0.0031 * * *$ & $-0.0041 * * *$ & $-0.0032 *$ & $-0.0047 * * *$ \\
\hline BMW 7-series & -0.0075 & $-0.0099 * * *$ & $-0.0067 * * *$ & $-0.0041 * * *$ & $-0.0092 * * *$ & -0.0006 \\
\hline Citroën $\mathrm{AX} / \mathrm{saxo} / \mathrm{C} 2$ & $0.0059 *$ & 0.0018 & $-0.0027 *$ & $-0.0069 * * *$ & -0.0036 & -0.0093 \\
\hline Citroën ZX/ Xsara/C4 & $0.0098 * * *$ & $0.0039^{*}$ & -0.0008 & $-0.0058 * * *$ & -0.0045 & -0.0074 \\
\hline Citroën Xantia/C5 & $0.0042 *$ & $0.0030^{*}$ & -0.0010 & $-0.0045 * *$ & 0.0007 & $-0.0091 * *$ \\
\hline Fiat Cinq./Seicento/Panda & $0.0138^{*}$ & $0.0127 * * *$ & 0.0023 & $-0.0055^{*}$ & $0.0127 * * *$ & -0.0168 *** \\
\hline Fiat Punto & 0.0067 & $0.0056 * *$ & -0.0009 & $-0.0060 * * *$ & -0.0018 & -0.0024 \\
\hline Fiat Bravo/Stilo & $0.0213 * * *$ & $0.0077^{*}$ & -0.0009 & $-0.0098 * * *$ & -0.0095 & -0.0069 \\
\hline Ford Fiesta & 0.0086 & 0.0002 & $-0.0026^{*}$ & $-0.0049 * * *$ & -0.0045 & -0.0009 \\
\hline Ford Escort/Focus & 0.0088 & 0.0032 & -0.0008 & $-0.0041 * * *$ & -0.0017 & -0.0030 \\
\hline Ford Mondeo & $0.0114 * *$ & 0.0003 & $-0.0031 * * *$ & $-0.0069 * * *$ & $-0.0094 * * *$ & $-0.0044 * *$ \\
\hline Honda Civic & $0.0107 * * *$ & 0.0001 & $-0.0035 * * *$ & $-0.0088 * * *$ & $-0.0144 * * *$ & -0.0037 \\
\hline Honda Accord & $0.0079 * *$ & $0.0049 * * *$ & -0.0012 & $-0.0063 * * *$ & -0.0004 & $-0.0088 * * *$ \\
\hline Mazda 3-series & 0.0120 *** & $0.0057 *$ & $-0.0036^{*}$ & $-0.0112 * * *$ & -0.0036 & $-0.0108 * * *$ \\
\hline Mercedes 180/c180 & -0.0026 & -0.0016 & $-0.0014 * * *$ & $-0.0011 * *$ & -0.0013 & -0.0005 \\
\hline Mercedes 200E/E220 & -0.0086 *** & $-0.0040 * * *$ & $-0.0030 * * *$ & $-0.0016 * * *$ & 0.0000 & -0.0021 \\
\hline Mercedes $320 \mathrm{~S} / \mathrm{S} 350$ & -0.0048 & $-0.0047 * * *$ & $-0.0036 * * *$ & $-0.0033 * * *$ & $-0.0059 * *$ & $-0.0018 *$ \\
\hline Nissan Micra & 0.0020 & 0.0017 & -0.0012 & -0.0018 & $0.0067 * *$ & $-0.0073 * * *$ \\
\hline Nissan Sunny/Almera & $0.0111 * *$ & $0.0071 * * *$ & -0.0002 & $-0.0055 * * *$ & 0.0040 & $-0.0104 * * *$ \\
\hline Nissan Primera & 0.0055 & 0.0029 & -0.0009 & $-0.0034 * * *$ & 0.0015 & $-0.0045 * * *$ \\
\hline Opel Corsa & 0.0025 & 0.0007 & $-0.0037 * * *$ & -0.0060 *** & 0.0008 & $-0.0073^{* *}$ \\
\hline Opel Astra & $0.0060^{*}$ & 0.0024 & $-0.0020 *$ & $-0.0051 * * *$ & -0.0007 & $-0.0054 *$ \\
\hline Opel Vectra & 0.0060 & 0.0008 & $-0.0019 * *$ & $-0.0034 * * *$ & 0.0001 & $-0.0070 * * *$ \\
\hline Opel Omega/Signum & 0.0008 & $0.0025 * *$ & $-0.0022 * *$ & $-0.0054 * * *$ & 0.0021 & $-0.0105 * * *$ \\
\hline Peugeot 306/307 & $0.0127 * * *$ & $0.0063 * * *$ & -0.0012 & $-0.0071 * * *$ & 0.0010 & $-0.0112 * * *$ \\
\hline Peugeot 405/406/407 & 0.0019 & $0.0052 * * *$ & 0.0011 & -0.0012 & 0.0097 & $-0.0118 * * *$ \\
\hline Renault Clio & $0.0068 * *$ & $0.0049 * * *$ & 0.0004 & $-0.0032 * * *$ & 0.0004 & -0.0021 \\
\hline Renault 19/Megane & 0.0025 & 0.0023 & -0.0014 & $-0.0033 * *$ & 0.0033 & $-0.0057 * *$ \\
\hline Renault Laguna & 0.0019 & $0.0049 * *$ & -0.0014 & $-0.0053 * *$ & 0.0055 & $-0.0066 *$ \\
\hline Range Rover & -0.0068 & -0.0059 & $-0.0102 * * *$ & $-0.0185^{* * *}$ & -0.0313 & $-0.0047 * * *$ \\
\hline Seat Ibiza & $0.0087 *$ & 0.0000 & $-0.0021 * *$ & $-0.0056 * * *$ & $-0.0105 * * *$ & -0.0011 \\
\hline Seat Cordoba & $0.0100 * *$ & 0.0017 & -0.0016 & $-0.0052 * * *$ & $-0.0076^{* *}$ & 0.0003 \\
\hline Seat Toledo & $0.0113 * *$ & $0.0052 * *$ & -0.0008 & $-0.0060 * * *$ & -0.0029 & -0.0029 \\
\hline Toyota Starlet/Yaris & 0.0029 & -0.0035 & $-0.0047 * * *$ & $-0.0056^{* * * *}$ & $-0.0071 *$ & $-0.0017 *$ \\
\hline Toyota Corola & 0.0073 & 0.0029 & -0.0010 & $-0.0050 * * *$ & -0.0046 & -0.0016 \\
\hline Toyota Carina/Avensis & $0.0050 *$ & $0.0036^{*}$ & -0.0017 & $-0.0065 * * *$ & -0.0030 & $-0.0068 * * *$ \\
\hline Volvo 440/s40 & 0.0025 & 0.0002 & 0.0010 & 0.0007 & -0.0039 & 0.0028 \\
\hline VW Polo & $0.0106 * * *$ & -0.0014 & $-0.0047 * * *$ & $-0.0081 * * *$ & $-0.0150 * * *$ & 0.0017 \\
\hline VW Golf & $0.0108 * * *$ & 0.0016 & $-0.0022 * *$ & $-0.0068 * * *$ & $-0.0096 * * *$ & $-0.0028 * *$ \\
\hline VW Passat & $0.0122 * * *$ & -0.0001 & $-0.0024 * *$ & $-0.0056 * * *$ & $-0.0108 * * *$ & -0.0006 \\
\hline
\end{tabular}

Notes: * significant at 10\%; ** significant at 5\%; *** significant at $1 \%$. Each trend is the coefficient estimate of biannual price dispersion (measured as the coefficient of variation) regressed on a time trend. The regressions include a constant. Group B includes: Finland, Greece, Ireland, Italy, Portugal, Spain, Sweden, and the United Kingdom 


\title{
PRICE CONVERGENCE IN THE EUROPEAN CAR MARKET
}

\author{
Salvador Gil-Pareja* \\ (Universidad de Valencia) \\ Simón Sosvilla-Rivero \\ (FEDEA and Universidad Complutense de Madrid)
}

Version: March 2006

\begin{abstract}
This paper examines price convergence in the European Union car market over the period 1995-2005. We find that there is a clear evidence of price convergence among the EU15 countries, but not before 1999. Moreover, countries of the Economic and Monetary Union (EMU) started convergence previously to the EU15 as a whole. Finally, exchange rate changes have significantly contributed to price dispersion over time across countries. The results provide significant evidence that trade liberalization and the EMU have enhanced the process of regional integration in the European automobile industry, even though there is room for further measures to promote integration.
\end{abstract}

JEL classification numbers: F15; F30.

Key Words: Market integration, Automobiles, European Union, Euro, Exchange rates.

\footnotetext{
*Corresponding author: Salvador Gil Pareja, Departamento de Estructura Económica, Av. de los Naranjos s/n, Valencia CP 46022, Spain; email: Salvador.Gil-Pareja@uv.es. We would like to thank José Antonio Martínez Serrano, Oscar Bajo Rubio, an anonymous referee, and the editor for valuable comments and suggestions. We also thank Frank Verboven and Pinelopi Goldberg for sending us the price data in Denmark, Finland and Greece prior to 1999. Salvador Gil-Pareja acknowledges financial support from Ministerio de Ciencia y Tecnología (project SEC2003-05836/ECO). Simón Sosvilla-Rivero gratefully acknowledges the financial support by Ministerio de Ciencia y Tecnologia grant SEJ2005-09094/ECON.
} 


\section{Introduction}

Over the past decades, important steps have been taken in the European Union (EU) to integrate markets. One of the expected effects of the process of market integration in Europe is price convergence. This hope relies on the argument that the elimination of administrative and technical barriers to trade, as a result of the Single Market Project, and the recent adoption of the euro reduce the potential for price discrimination across member States by bolstering cross-border trade and price transparency.

There is a growing number of papers on the issues of price and inflation convergence among the EU member States (see, e. g., Camarero et al., 2000; Rogers, 2001; Rogers et al., 2001; Gámez-Amián and Morales-Zumaquero, 2002; SosvillaRivero and Gil-Pareja, 2004; Chen, 2004; or Gil-Pareja and Sosvilla-Rivero, 2004) and the Economic and Monetary Union (EMU) countries (see, e. g., Parsley and Wei, 2001; Rogers, 2001 and 2002; Baye et al., 2003, Mathä, 2003; Gajewski and Kowalski, 2004; Isgut, 2004; Engel and Rogers, 2004; or Allington et al., 2005). Altogether, these studies show evidence of price convergence for the EU whereas the evidence for the EMU is mixed. ${ }^{1}$

One market that has attracted particular interest is the European car market. A number of studies have focused on price convergence in this market. Gaulier and Haller (2000), using aggregate prices constructed as averages of car prices, do not find evidence of convergence over the 1993-1999 period for 10 EU countries, and conclude that exchange rate fluctuations explain a large share of the price dispersion dynamics. Lutz (2004a) finds that there has been no tendency for average car price differentials to

\footnotetext{
${ }^{1}$ A recent and excellent review of the literature about price dispersion in the EU and, especially, in EMU countries is offered by Allington et al (2005).
} 
decline during 1993-1998 on a sample of 12 EU member States. Moreover, he shows that deviations from the law of one price are mainly related to factors that affect the cost of arbitrage activities between markets. Lutz (2004b) employs a difference-in-difference approach to prices of 17 car models in 12 EU countries over the 1995-2001 period, and finds that EMU has not let to a widespread narrowing of price dispersion during the first three years. Goldberg and Verboven (2001) document and explain car price dispersion using data for approximately 150 models, five markets (Belgium, France, Germany, Italy, and the UK) and 14 years (1980-1993). They find substantial year-to-year volatility in the car price data that is to a large extent accounted by exchange rate fluctuations and the incomplete response of local currency prices to these fluctuations. ${ }^{2}$ Goldberg and Verboven (2004) find that absolute price differentials declined over the 1993-2003 period and, using a difference-in-difference approach, are able to derive an estimate of the monetary union's contribution to this decline of approximately $1-2 \%$. In a later paper, Goldberg and Verboven (2005) document, for the cited five countries, that cross-country price differentials for the new cars declined over the $1970-2000$ period, and attribute this decline to the European integration process of the 1990s.

The automobile industry provides a good opportunity for studying price convergence within both EU and EMU countries. On the one hand, cross-country price differentials in the automobile industry are an important source of concern under the European Commission's competition policy. On the other hand, since May 1993, the European Commission publishes, twice a year, car price surveys for most car models sold in the EU. These surveys represent one of the rare comprehensive public sources of information on product prices in the EU at such detailed level.

\footnotetext{
${ }^{2}$ For the automobile industry several studies have found an incomplete degree of exchange rate passthrough to import prices (Gross and Schmitt, 1996; Gron and Swenson, 1996; Feenstra, et al., 1996), and
} 
The aim of this paper is to examine whether the EU15 and the eurozone car markets have become more integrated, using as a metric the dispersion of prices net of taxes. Several studies of cross-country price behaviour in the European car market have used the European Commission surveys. Our study extends those works by using a different approach to examining this data set and by incorporating the most recent information. In particular, unlike previous studies we use the concept of $\sigma$-convergence to individual car models and our data set includes 13 post-EMU and 7 post-euro price surveys.

Moreover, since the European Monetary System (EMS) represented an intermediary step to the EMU, fostering economic integration and economic policy coordination in the EU, we devote particular attention to the convergence of prices experienced by countries whose currencies participated in the core of EMS. In this regard, it has been claimed that international trade in a regime of relatively fixed exchange rates such as that established by EMS would result in price convergence. Therefore, by analysing price dispersion among EMS countries with different degrees of exchange rate stability we hope to shed new light on the success of this exchange rate agreement in terms of imposing price discipline among its members.

The paper is organised as follows. Section 2 shortly discusses economic incentives and institutions generating deviations from the law of one price. Section 3 presents the data and Section 4 sets out the empirical findings. Finally, Section 5 offers some concluding remarks.

\section{The law of one price and the European car market}


Much work in international economics has focused on testing the validity of the law of one price across countries. There are two versions of the law of one price: the absolute and the relative versions. The absolute version states that, in the absence of transfer costs and under competitive conditions, identical tradable goods priced in a common currency should be equal across countries. The intuition is that international arbitrage should work until prices are aligned. In its relative form, the law of one price asserts that common currency prices for a particular product should change in the same way over time in different countries and, therefore, it is compatible with the existence of a stable price differential across markets.

Most of the empirical literature on the law of one price examines the validity of its relative version for two main reasons. First, arbitrage is not costless. Trading between locations itself has costs (such as transportation costs and trade barriers), so prices are very unlikely to be identical across locations. However, these costs may give rise to a stable price differential across markets. Second, the preference for testing the relative version is a consequence of data limitations rather than research interest. Typically, the data employed in price comparisons is in the form of price indices in different countries whose levels are arbitrary. However, in this paper, the price information is based on recommended retail prices of specific car models. The prices used are in ECUs/euros, net of taxes and have been adjusted for equipment differences. It allows us to focus on the analysis of the convergence to the absolute version of the law of one price.

Two conditions are necessary for the existence of international price differences beyond transfer costs. First, firms must have market power as well as some profit incentives to set different prices in different countries. Different demand elasticities, import quotas, or an incomplete pass-through (of taxes or exchange rates) are the most frequently studied sources of markup differences across countries in the European car 
market and, therefore, of international price discrimination. Second, firms must be able to prevent arbitrage. Traditionally, several non-tariff barriers, such as the type approval or the national registration, have contributed to keep European car markets geographically segmented. Despite the removal of a great part of the barriers within the EU in 1993, some obstacles to cross-border trade have remained since then. In particular, during the sample period the major obstacle to cross-border trade stems from the selective and exclusive distribution system authorised by Regulation 123/85 (from 1985 to 1995 ) and 1475/95 (from 1995 up to 30 September 2002). This distribution system aimed to restrict sales of new cars in the EU to dealers chosen by manufacturers, becoming very difficult for independent wholesalers to buy cars in bulk in one country and resell them in another. An important liberalization of the distribution system was introduced in 2002 with transitions periods of at least one year. ${ }^{3}$

\section{Data}

The price data used in this study come from the biannual surveys of car price differentials between EU member States carried out by the European Commission since 1993. The methodology used has remained the same for all the surveys over the period 1993-2005. About 17 European and 8 Japanese manufacturers submit the recommended retail prices on 1 May and 1 November of each year of their top-selling products. The number of car models included in these surveys ranges from 72 to 91 . The prices are adjusted for equipment differences and are given in local currency and in ECU/euros, both before and after tax. It shall be noted that actual retail prices may differ from recommended list prices, as dealers are free to set their own prices. In particular, the

\footnotetext{
${ }^{3}$ See Goldberg and Verboven (2004) for a detailed discussion about both the profit incentives for price discrimination in the car market and the European institutions that have made international price discrimination feasible in this industry.
} 
data set on pre-tax list prices used in this study consists of the surveys conducted over the period from May 1995 to May 2005 (21 bi-annual surveys). The countries covered are all the EU-15 member States. ${ }^{4}$ The car models considered are those with appropriate data in the range of countries and periods for the purposes of this paper. As a result, we have selected a sample of 45 models.

\section{Empirical results}

There are various ways of measuring price dispersion, for example, the range of minimum price to maximum price, the ratio of maximum price to minimum price, the ratio of maximum price to mean price, the standard deviation, or the coefficient of variation. We use the coefficient of variation (the ratio of the standard deviation to the mean) as a measure of price dispersion because it has advantages over the cited alternative measures. The coefficient of variation is invariable to changes of scale, which is useful for comparing price dispersion across products or, for a given product, price dispersion over time. This affords an advantage with respect to the range and the standard deviation. Moreover, although the range and max-min ratio provide a measure of the total spread of the data, they only take into account the two extreme values of the data, and, therefore, they are susceptible to considerable distortion if there is an unusual extreme observation. Similarly, the max-mean ratio only considers all the observations in the computation of the mean, in contrast to the coefficient of variation, which takes into account each of the data observations in both the numerator (which measures the average spread around the mean) and the denominator (the mean).

\footnotetext{
${ }^{4}$ The pre-tax car prices are available since May 1993 for 10 European countries (Belgium, France, Germany, Ireland, Italy, Luxembourg, the Netherlands, Portugal, Spain, the United Kingdom). Pre-tax prices for Denmark and Greece are available since November 1994, while for Austria, Finland and Sweden they are available since May 1995. Data for the ten new members of the EU (Cyprus, Czech
} 
For the purpose of assessing price convergence in the European car market we use the concept of $\sigma$-convergence. ${ }^{5}$ Thus, to measure the degree of price convergence for each car model, first, we calculate the coefficient of price variation for each bi-annual survey across the corresponding set of European markets. Then, the time series of coefficients of variation for each car model $\left(\mathrm{CV}_{\mathrm{t}}\right)$ are regressed on a constant and a linear time trend (TIME):

$$
C V_{t}=\alpha+\sigma T I M E+u_{t}
$$

where $\alpha$ and $\sigma$ are parameters to be estimated, and $u_{t}$ is the error term. If price dispersion declined steadily, we would expect the regression to yield a negative and statistically significant coefficient for the linear time trend.

Tables 1-4 present the results for the 45 car models considered in four different samples of countries. The first table presents the results for EU15. Table 2 focuses on EMU11 countries. Table 3 ("group A") examines price convergence for EU15 countries whose currencies participated continuously in the Exchange Rate Mechanism (ERM) of the EMS from the outset maintaining broadly stable bilateral exchange rates among themselves (Austria, Belgium, Denmark, France, Germany, Luxembourg, and the Netherlands). Finally, Table 4 (“group B”) includes EU15 countries whose currencies showed considerable fluctuations in value relative to the German mark (Finland, Greece, Ireland, Italy, Portugal, Spain, Sweden, and the United Kingdom). ${ }^{6}$ Each table reports the estimated coefficients on the linear trend in the coefficients of variation for six

Republic, Estonia, Latvia, Lithuania, Hungary, Malta, Poland, Slovakia, Slovenia) are not considered given that they are only available since May 2004.

${ }_{5}^{5} \sigma$-convergence occurs if the cross-sectional dispersion of a variable decreases over time.

${ }^{6}$ It is interesting to note that these two groups roughly correspond to the distinction made by the European Commission (1995) between those countries whose currencies continuously participated in the ERM from its inception maintaining broadly stable bilateral exchange rates among themselves over the sample period, and those countries whose currencies either entered the ERM later or suspended its participation in the ERM, as well as fluctuating in value to a great extent relative to the Deutschmark. These two groups are also basically the same found in Jacquemin and Sapir (1996), applying multivariate analysis 
periods. The first three periods (columns 1 to 3) start in 1995, ending in 1998 (the year before irrevocable exchange rates among EMU countries are fixed), 2001 (the year prior to the introduction of the euro) and 2005 (the last year in the sample), respectively. In column 4 we analyse price convergence over the period 1998-2005. Finally, in columns 5 and 6 this recent period is divided into two which allows us to distinguish the first years of the EMU (when the euro did not circulated) and from that time onwards.

In general, the evidence of price dispersion trends varies both according to the sample of countries and the periods considered. As can be observed in Table 1, during the period 1995-1998 about only half of the estimates are negative and just 5 of them are statistically significant at the $10 \%$ level. Furthermore, 4 of the 21 positive coefficients reach the statistical significance. Therefore, it is not surprising that the average coefficient of variation in $1995(0.1008)$ be very similar to that found in $1998(0.1002)$. One fact that may help to explain the scarce evidence of convergence during this period is the presence of an incomplete pass-through of exchange rates to prices. As noted before, in the automobile industry, several studies have found an incomplete degree of exchange rate pass-through to import prices (Gross and Schmitt, 1996; Gron and Swenson, 1996; Feenstra, et al., 1996) and evidence of pricing to market behaviour (Gagnon and Knetter, 1995; Gil-Pareja, 2001; Gil-Pareja, 2003). ${ }^{7}$ Extending the period to 2001 , we find an increase in the number of negative and statistically significant coefficients, but the distribution of positive and negative coefficients remains unchanged. However, when we add to the sample period data from 2002 onwards, the

techniques (i.e., principal components and cluster analysis) to a wide set of structural and macroeconomic indicators, to form an homogeneous group of countries.

${ }^{7}$ In particular, Gil-Pareja (2003) investigates pricing to market behaviour in European car markets during the period 1993-1998 using also the bi-annual data provided by the European Commission surveys. He concludes that local currency price stability is a strong and pervasive phenomenon across products that is consequence, at least in part, of the existence of market segmentation and international price discrimination, despite the completion of the single market programme in 1993. 
existence of price convergence is evident: 44 out of 45 coefficients are negative and $89 \%$ of them are statistically significant. A similar result emerges when we analyse price convergence over the period 1998-2005, for which the estimated coefficients for the linear time trend is negative in all the cases and they are statistically significant at the $1 \%$ level in $87 \%$ of them. Splitting this period into two we find a clear evidence of price convergence in both sub-periods although it is stronger after 2001 than from 1998 to 2001.

In comparison with the EU15, the sample of EMU11 countries (Table 2) shows a remarkably greater number of negative coefficients during the periods 1995-1998 and 1995-2001 while for the full sample period the number of negative trends is slightly smaller. ${ }^{8}$ This result suggests that EMU countries started convergence previously to the EU as a whole, which, as noted before, does not show evidence of convergence before 1999. Moreover, the study of price convergence after that year provides broadly the same picture than for the EU in its entity. Nonetheless, two comments are in order. First, there is more evidence of convergence among the EU15 than among EMU countries. This might be expected since in 1998 price dispersion was considerably smaller across EMU countries (0.0669) than across EU member States (0.1002). Second, as was expected the evidence of price convergence is stronger in the post-euro period. ${ }^{9}$ The inclusion of Greece among the set of EMU countries leads to the same conclusions. ${ }^{10}$

Another important issue that is an aim of this paper is the analysis of the impact of exchange rates on convergence patterns. To this end, we study whether EU15

\footnotetext{
${ }^{8}$ The result for the period 1995-2001 contrast with Lutz (2004b)'s conclusions on a sample of 17 car models.

${ }^{9}$ Friberg (2001) provides theoretical support to the notion that the single currency should reduce the potential for price discrimination across participating countries. He shows that a monetary union promotes market integration by reducing the option value of segmenting markets.

${ }^{10}$ To economise on space, we do not report the results including Greece among the set of EMU countries, but they are available from the authors upon request.
} 
countries with relatively stable and credible exchange rates prior to the EMU (group A) showed a stronger tendency towards convergence than countries with relatively volatile exchange rates (group B) ${ }^{11}$. In the stable currencies group (Table 3), a small majority, 26 out of 45 , cases show a declining trend over the period 1995-1998, although only 10 of them are statistically significant at the $10 \%$ level. However, in group B (Table 4 ) only 6 estimates are negative and only one of them is statistically significant at conventional levels. In contrast, in the last group 24 out of 39 positive coefficients reach the statistical significance at the $10 \%$ level. ${ }^{12}$ The enlargement of the sample period to 2001 increases the number of significant trends towards lower price dispersion in both groups, even though it is worth noting that there is again more evidence of convergence in group A (with 19 negative and statistically significant estimates) and that, in group B, 16 of the 34 positive coefficients are statistically significant. When we extend the sample period to 2005 , we observe significantly lower price dispersion over time in 35 (23) car models in group A (B). Focussing attention on the period 1998-2005, the results indicate a significant trend toward lower dispersion in both samples, but in this case it is particularly intense in group B where it occurs in 42 models. As a result of these trends the average coefficient of variation in 2005 in group B $(0.0561)$ is slightly smaller than in group A (0.0577). Finally, it is worth noting that the evidence that emerges after splitting this period into two does not differ markedly in both groups of countries, being greater the number of negative trends in the post-euro period.

\section{Concluding remarks}

\footnotetext{
${ }^{11}$ See Ledesma-Rodríguez et al. (2005) for an empirical evaluation of the credibility of the commitment to maintain the exchange rate around a central parity in the ERM.

${ }^{12}$ Consistent with these trends, the average coefficient of variation in group A fall from 0.095 to 0.087 over the period 1995-1998, whereas, in group B, it increases from 0.079 to 0.108 over the same period.
} 
This paper has offered empirical evidence of price convergence in one market that has attracted special attention during the last decade: the European car market. In particular, we have examined whether the EU15 and the eurozone car markets have become more integrated, using a different approach to that employed in previous works (the concept of $\sigma$-convergence to individual car models) and incorporating the most recent information. We have also explored the effect of exchange rates on price convergence patterns by reviewing the experience of European countries that participated in the exchange rate stability zone with a high degree of confidence with respect to the commitment to maintain the exchange rate around a central parity against countries with relatively volatile exchange rates. Even though the differences in the samples of countries and periods considered in previous studies calls for caution in comparing the results, our findings are, in general, consistent with the evidence reported in the introductory material.

Overall, the evidence of price convergence varies both according to the sample of countries and the periods considered. In the EU as a whole there is no tendency for price dispersion to fall over the period 1995-1998. In contrast, from 1998 onwards the evidence of price convergence is pervasive and stronger once the euro has replaced the national currencies of the member countries than until 2001. If we restrict the sample to the EMU markets, we observe significant lower price dispersion over time since 1995. It suggests that EMU countries started convergence previously to the EU as a whole. However, the study of price convergence after irrevocable exchange rates were fixed provides broadly the same picture than for the EU in its entity, being the average coefficient of variation in 2005 slightly smaller in EMU countries (0.048 against 0.057 in the EU15). Finally, exchange rate movements over the period 1995-1998 has significantly contributed to price dispersion across countries. In particular, countries 
with relatively volatile exchange rates show a tendency towards a higher price dispersion over the period 1995-1998. However, from 1998 onwards there is a very clear evidence of lower price dispersion over time.

We view our results as evidence that the progress towards integration in Europe, and especially the formation of the EMU, has had visible effects on cross-country price dispersion in recent years even though price convergence has not yet been completed. The findings in this paper have important policy implications. First, the comparison between countries with different degrees of exchange rate stability, as well as the evidence that price differentials become smaller before across EMU countries than across EU15 members, has implications for the role of exchange rate policy on price dispersion. Both suggest that exchange rate stability has contributed to market integration beyond the role of other integration measures. Second, since the evidence of price convergence is stronger in the post-euro period not only for the EMU members but also for the EU15, the decline in price dispersion cannot be attributed, at least only, to the euro. Finally, to the extent that, despite the evidence of convergence, price differences across EMU countries remain significant after four years from the introduction of the euro, additional measures to promote integration (such as tax harmonization) are needed to achieve full integration of the European car markets.

\section{References}

Allington, N. F. B., Kattuman, P. A. and Waldmann, F. A. (2005): "One market, one money, one price?” International Journal of Central Banking 1, pp. 73-115.

Baye, M., Gatti, R., Kattuman, P. and Morgan, J. (2003): "Did the euro foster online price competition? Evidence from an International Price Comparison Site", Economic Inquiry 44, pp. 265-279. 
Camarero, M., Esteve, V. and Tamarit, C. (2000): "Price convergence of the peripheral European countries on the way to the EMU: A time series approach", Empirical Economics 25, pp. 149-168.

Chen, N. (2004): "The behaviour of relative prices in the European Union: A sectoral analysis", European Economic Review 48, pp. 1257-1286.

Engel, C. and Rogers, J. H. (2004): "European product market integration after the euro", Economic Policy 19, pp. 347-384.

European Commission (1995): "The impact of exchange rate movements on trade within the single market", European Economy, Reports and Studies, No. 4.

Feenstra, R. C., Gagnon, J. E. and Knetter, M. M. (1996): "Market share and exchange rate pass-through in world automobile trade", Journal of International Economics 40, pp. 187-207.

Friberg, R. (2001): “Two monies, two markets? Variability and the option to segment", Journal of International Economics 55, pp. 317-327.

Gagnon, J. E. and Knetter, M. M. (1995): "Markup adjustment and exchange rate fluctuations: evidence from panel data on automobile exports", Journal of International Money and Finance 14, pp. 289-310.

Gámez-Amián, C. and Morales-Zumaquero, A. (2002): Complete or partial inflation convergence in the EU?, Working paper E2002/09, Sevilla, CentrA.

Gajewski, P. and Kowalski, J. (2004): Price convergence in the European Union countries, Institute of Economics, University of Lodz, mimeo.

Gaullier, G. and Haller, S. (2000): "The convergence of automobile prices in the European Union: an empirical analysis for the period 1993-1999”, CEPII, Working Paper 2000-14. 
Gil-Pareja, S. (2001): "Pricing to market in European automobile exports to OECD countries: a panel data approach", Applied Economics 14, pp. 1845-1856.

Gil-Pareja, S. (2003): "Pricing to market behaviour in European car markets", European Economic Review 47, pp. 945-962.

Gil-Pareja, S. and Sosvilla-Rivero, S. (2004): "Export market integration in the European Union", Journal of Applied Economics 7, pp. 271-301.

Goldberg, P. K. and Verboven, F. (2001): "The evolution of price dispersion in the European car market", Review of Economic Studies 68, pp. 811-848.

Goldberg, P. K. and Verboven, F. (2004): “Cross-country price dispersion in the euro era: a case study of the European car market”, Economic Policy 19, pp. 483-521.

Goldberg, P. K. and Verboven, F. (2005): "Market integration and convergence to the law of one price: evidence from the European car market", Journal of International Economics 65, pp. 49-74.

Gron, A. and Swenson, D. L. (1996): "Incomplete exchange rate pass-through and imperfect competition: the effect of local production", American Economic Review 86, pp. 71-76.

Gross, D. G. and Schmitt, N. (1996): "Exchange rate pass-through and rivalry in the Swiss automobile market", Weltwirtschaftliches Archiv 132, pp. 278-303.

Isgut, I. (2004): “Common currencies and market integration across cities: How strong is the link?", in V. Alexander, J. Mélitz, and G. M. von Furstenberg (eds.), Monetary Unions and Hard Pegs: Effects on Trade, Financial Development, and Stability (Oxford: Oxford University Press), Chapter 8.

Jacquemin, A. and Sapir, A. (1996): "Is a European hard core credible? A statistical analysis", Kyklos 49, pp. 105-117. 
Ledesma-Rodríguez, F., Navarro-Ibáñez, M., Pérez-Rodríguez, J., and Sosvilla-Rivero, S. (2005): "Assessing the Credibility of a Target Zone: Evidence from the EMS", Applied Economics 37, pp. 2265-2287.

Lutz, M. (2004a): "Pricing in segmented markets, arbitrage barriers and the law of one price: evidence from the European car market", Review of International Economics 12 pp. $456-475$.

Lutz, M. (2004b): "Price convergence under the EMU? First estimates", in A. Deardoff (Ed.), Past, present, and future of European Union, Proceedings of the $13^{\text {th }}$ World Congress of the International Economic Association, Macmillan Press.

Mathä, T. Y. (2003): What of expect to the euro? Analysing price differences of individual products in Luxembourg and its surrounding regions, Banque Centrale du Luxembourg, Working Paper 8.

Parsley, D. C. and Wei, S. J. (2001): Limiting currency volatility to stimulate goods market integration, NBER Working Paper No. 8468.

Rogers, J. H. (2001): "Price level convergence, relative prices, and inflation in Europe", International Finance Discussion Paper 699, Board of Governors of the Federal Reserve System.

Rogers, J. H. (2002): "Monetary union, price level convergence, and inflation: How close is Europe to the United States?", forthcoming in Journal of Monetary Economics. Rogers, J. H., Hufbauer, G. C. and Wada, E. (2001): Price level convergence and inflation in Europe, Working Paper 01-1, Institute for International Economics.

Sosvilla-Rivero, S. and Gil-Pareja, S. (2004): "Price convergence in the European Union”, Applied Economics Letters 11, pp. 39-47. 
Table 1

Trends in Price Dispersion: Sample EU15

\begin{tabular}{|c|c|c|c|c|c|c|}
\hline Model & (1) & (2) & (3) & (4) & (5) & (6) \\
\hline & $1995-1998$ & $1995-2001$ & $1995-2005$ & $1998-2005$ & $1998-2001$ & $2001-2005$ \\
\hline Alfa 145/147 & $0.0099 * *$ & 0.0030 & -0.0018 & $-0.0063 * * *$ & $-0.0068 * *$ & $-0.0037 * * *$ \\
\hline Alfa $155 / 156$ & 0.0043 & $0.0020 * *$ & $-0.0029 * * *$ & $-0.0059 * * *$ & -0.0011 & -0.0058 \\
\hline Audi A4 & $-0.0026 * *$ & $-0.0036 * * *$ & $-0.0042 * * *$ & $-0.0045^{* * *}$ & $-0.0035 * *$ & $-0.0051 * * *$ \\
\hline Audi A6 & $0.0031 *$ & $-0.0017^{*}$ & $-0.0026^{* * *}$ & $-0.0039 * * *$ & $-0.0043^{*}$ & $-00064 * * *$ \\
\hline BMW 3-series & -0.0013 & $-0.0014 *$ & $-0.0024 * * *$ & $-0.0028 * * *$ & -0.0008 & $-0.0037 * * *$ \\
\hline BMW 5-series & -0.005 & $-0.0024 *$ & $-0.0031 * * *$ & $-0.0034 * * *$ & $-0.0025^{*}$ & $-0.0043 * * *$ \\
\hline BMW 7-series & -0.0013 & $-0.0056^{*}$ & $-0.0043 * * *$ & $-0.0031 * * *$ & $-0.0058 * * *$ & -0.00012 \\
\hline Citroën AX/saxo/C2 & -0.0026 & -0.0004 & $-0.0025 * * *$ & $-0.0044 * * *$ & -0.0014 & $-0.0060 *$ \\
\hline Citroën ZX/ Xsara/C4 & -0.0028 & 0.0020 & -0.0015 & $-0.0044 * * *$ & 0.0017 & $-0.0090 * * *$ \\
\hline Citroën Xantia/C5 & $-0.0060 * * *$ & 0.0012 & $-0.0014^{*}$ & $-0.0030 * *$ & 0.0032 & $-0.0054 * * *$ \\
\hline Fiat Cinq./Seicento/Panda & -0.0036 & $0.0038 * *$ & -0.0001 & -0.0011 & $0.0123 * * *$ & $-0.0092 * * *$ \\
\hline Fiat Punto & 0.0033 & 0.0021 & $-0.0018^{*}$ & $-0.0047 * * *$ & -0.0021 & -0.0018 \\
\hline Fiat Bravo/Stilo & $0.0078 * *$ & 0.0030 & -0.0016 & $-0.0065 * * *$ & -0.0057 & -0.0050 \\
\hline Ford Fiesta & 0.0043 & -0.0018 & $-0.0029 * * *$ & $-0.0037 * * *$ & $-0.0046 * *$ & 0.0000 \\
\hline Ford Escort/Focus & 0.0019 & -0.0007 & $-0.0021 * * *$ & $-0.0029 * * *$ & -0.0016 & $-0.0032 * * *$ \\
\hline Ford Mondeo & 0.0030 & -0.0016 & $-0.0033 * * *$ & $-0.0052 * * *$ & $-0.0055 * * *$ & $-0.0059 * * *$ \\
\hline Honda Civic & 0.0040 & -0.0017 & $-0.0030 * * *$ & $-0.0054 * * *$ & $-0.0097 * * *$ & -0.0018 \\
\hline Honda Accord & -0.0017 & 0.0008 & $-0.0019 * * *$ & $-0.0038 * * *$ & 0.0005 & $-0.0067 * * *$ \\
\hline Mazda 3-series & $0.0061 * * *$ & 0.0027 & $-0.0029 * *$ & $-0.0079 * * *$ & -0.0035 & $-0.0076 * * *$ \\
\hline Mercedes 180/c180 & $-0.0039 *$ & $-0.0024 * * *$ & $-0.0016^{* * *}$ & $-0.0010^{* * *}$ & $-0.0018^{*}$ & -0.0004 \\
\hline Mercedes 200E/E220 & $-0.0055^{* *}$ & $-0.0032 * * *$ & $-0.0027 * * *$ & $-0.0017 * * *$ & -0.0004 & $-0.0017 *$ \\
\hline Mercedes 320S/S350 & -0.0021 & $-0.0038 * * *$ & $-0.0031 * * *$ & $-0.0329 * * *$ & $-0.0052 * *$ & $-0.0011 *$ \\
\hline Nissan Micra & -0.0018 & -0.0012 & $-0.0019 * * *$ & -0.0014 & $0.0027 *$ & $-0.0039 * *$ \\
\hline Nissan Sunny/Almera & 0.0028 & $0.0021 *$ & $-0.0014^{*}$ & $-0.0034 * * *$ & 0.0026 & $-0.0064 * * *$ \\
\hline Nissan Primera & -0.0007 & 0.0012 & $-0.0014 * *$ & $-0.0024 * *$ & $0.0044^{*}$ & $-0.0053 * * *$ \\
\hline Opel Corsa & -0.0010 & -0.0004 & $-0.0034 * * *$ & $-0.0047 * * *$ & 0.0015 & $-0.0067 * * *$ \\
\hline Opel Astra & 0.0022 & 0.0009 & $-0.0018 * * *$ & $-0.0038 * * *$ & -0.0012 & $-0.0041 *$ \\
\hline Opel Vectra & 0.0017 & 0.0005 & $-0.0023 * * *$ & $-0.0038 * * *$ & 0.0012 & $-0.0061 * *$ \\
\hline Opel Omega/Signum & -0.0008 & 0.0006 & $-0.0022 * * *$ & $-0.0042 * * *$ & 0.0005 & $-0.0072 * * *$ \\
\hline Peugeot 306/307 & 0.0030 & $0.0034 * * *$ & -0.0007 & $-0.0039 * * *$ & 0.0021 & $-0.0076^{* * *}$ \\
\hline Peugeot 405/406/407 & $-0.0043 *$ & $0.0033 * * *$ & 0.0012 & -0.0002 & $0.0072 * * *$ & $-0.0097 *$ \\
\hline Renault Clio & 0.0020 & $0.0016^{*}$ & $-0.0014 * *$ & $-0.0037 * * *$ & -0.0002 & $-0.0039 * *$ \\
\hline Renault 19/Megane & 0.0030 & 0.0008 & $-0.0020 * * *$ & $-0.0039 * * *$ & 0.0006 & $-0.0062 *$ \\
\hline Renault Laguna & -0.0006 & 0.0021 & $-0.0018 * *$ & $-0.0043 * * *$ & 0.0027 & $-0.0056 * * *$ \\
\hline Range Rover & -0.0065 & -0.0043 & $-0.0077 * * *$ & $-0.0139 * * *$ & -0.0224 & $-0.0039 * * *$ \\
\hline Seat Ibiza & -0.0014 & $-0.0026^{*}$ & $-0.0034 * * *$ & $-0.0048 * * *$ & $-0.0062 *$ & -0.0017 \\
\hline Seat Cordoba & 0.0012 & -0.0014 & $-0.0034 * * *$ & $-0.0053 * * *$ & $-0.0057^{*}$ & -0.0022 \\
\hline Seat Toledo & -0.0015 & -0.0009 & $-0.0027 * * *$ & $-0.0040 * * *$ & -0.0017 & $-0.0030 * * *$ \\
\hline Toyota Starlet/Yaris & -0.0005 & $-0.0034^{*}$ & $-0.0039 * * *$ & $-0.0040 * * *$ & $-0.0037^{*}$ & $-0.0028 * * *$ \\
\hline Toyota Corola & -0.0007 & -0.0012 & $-0.0026^{* * *}$ & $-0.0041 * * *$ & -0.0033 & -0.0037 \\
\hline Toyota Carina/Avensis & 0.0007 & 0.0006 & $-0.0023 * * *$ & $-0.0049 * * *$ & -0.0027 & $-0.0057 * * *$ \\
\hline Volvo $440 / \mathrm{s} 40$ & -0.0048 & $-0.0032 * * *$ & $-0.0017 * *$ & -0.0008 & -0.0022 & -0.0016 \\
\hline VW Polo & 0.0024 & $-0.0038 * * *$ & $-0.0037 * * *$ & $-0.0046 * * *$ & $-0.0103 * * *$ & 0.0011 \\
\hline VW Golf & $0.0067 * * *$ & 0.0006 & $-0.0014 * *$ & $-0.0040 * * *$ & $-0.0062 * * *$ & $-0.0014 * * *$ \\
\hline VW Passat & 0.0045 & -0.0017 & $-0.0021 * * *$ & $-0.0031 * * *$ & $-0.0066 * * *$ & -0.0002 \\
\hline
\end{tabular}

Notes: * significant at $10 \%$; ** significant at $5 \% ; * * *$ significant at $1 \%$. Each trend is the coefficient estimate of biannual price dispersion (measured as the coefficient of variation) regressed on a time trend. The regressions include a constant. 
Table 2

Trends in Price Dispersion: Sample EMU11

\begin{tabular}{|c|c|c|c|c|c|c|}
\hline Model & (1) & (2) & (3) & (4) & (5) & (6) \\
\hline & 1995-1998 & $1995-2001$ & $1995-2005$ & $1998-2005$ & $1998-2001$ & $2001-2005$ \\
\hline Alfa 145/147 & -0.0011 & -0.0003 & $-0.0013 *$ & $-0.0033 * * *$ & $-0.0062 *$ & -0.0009 \\
\hline Alfa $155 / 156$ & $-0.0059 * * *$ & -0.0008 & -0.0010 & -0.0002 & $0.0024 *$ & 0.0004 \\
\hline Audi A4 & $-0.0020 * *$ & $-0.0056 * * *$ & $-0.0040 * * *$ & $-0.0031 * * *$ & $-0.0070 * *$ & -0.0006 \\
\hline Audi A6 & 0.0025 & $-0.0035 * * *$ & $-0.0028 * * *$ & $-0.0026 * *$ & -0.0050 & $-0.0042 * * *$ \\
\hline BMW 3-series & -0.0008 & -0.0009 & $-0.0011 * * *$ & $-0.0014 * * *$ & $-0.0014 * *$ & $-0.0015 *$ \\
\hline BMW 5-series & -0.0022 & $-0.0015^{*}$ & $-0.0019 * * *$ & $-0.0025 * * *$ & $-0.0019 * * *$ & $-0.0032 * * *$ \\
\hline BMW 7-series & -0.0019 & $-0.0043 * * *$ & $-0.0042 * * *$ & $-0.0047 * * *$ & $-0.0073 * * *$ & -0.0024 \\
\hline Citroën AX/saxo/C2 & $-0.0076 * * *$ & $-0.0063 * * *$ & $-0.0033 * * *$ & $-0.0016^{*}$ & $-0.0066 * * *$ & 0.0009 \\
\hline Citroën ZX/ Xsara/C4 & $-0.0067 * * *$ & $-0.0026 * * *$ & $-0.0025 * * *$ & $-0.0024 * * *$ & -0.0010 & $-0.0029 * * *$ \\
\hline Citroën Xantia/C5 & $-0.0062 * * *$ & $-0.0020 * *$ & $-0.0031 * * *$ & $-0.0036 * * *$ & -0.0002 & $-0.0048 * * *$ \\
\hline Fiat Cinq./Seicento/Panda & $-0.0082 * * *$ & -0.0000 & -0.0004 & 0.0005 & $0.0071 * * *$ & $-0.0052 * * *$ \\
\hline Fiat Punto & $-0.0081 * * *$ & $-0.0032 * * *$ & $-0.0014 * * *$ & 0.0004 & 0.0007 & 0.0003 \\
\hline Fiat Bravo/Stilo & -0.0013 & -0.0016 & -0.0006 & 0.0002 & -0.0017 & 0.0006 \\
\hline Ford Fiesta & 0.0015 & $-0.0049 * * *$ & $-0.0034 * * *$ & $-0.0021 * *$ & $-0.0061 * * *$ & 0.0009 \\
\hline Ford Escort/Focus & $-0.0036^{*}$ & $-0.0027 * * *$ & -0.0007 & 0.0010 & -0.0006 & -0.0002 \\
\hline Ford Mondeo & -0.0017 & $-0.0033 * *$ & $-0.0026^{* * *}$ & $-0.0020 * * *$ & -0.0029 & $-0.0029 * * *$ \\
\hline Honda Civic & -0.0017 & $-0.0024 * * *$ & $-0.0016^{* * * *}$ & $-0.0018 * * *$ & $-0.0044 * * *$ & -0.0006 \\
\hline Honda Accord & $\begin{array}{l}-0.0011 \\
\end{array}$ & 0.0000 & $\begin{array}{l}-0.0006 \\
\end{array}$ & $\begin{array}{l}-0.0009 \\
\end{array}$ & 0.0019 & $-0.0055 * * *$ \\
\hline Mazda 3-series & 0.0031 & 0.0014 & -0.00011 & $-0.0032 * * *$ & -0.0005 & $-0.0038 * *$ \\
\hline Mercedes 180/c180 & $-0.0056 * * *$ & $-0.0027 * * *$ & $-0.0018 * * *$ & $-0.0011 * * *$ & -0.0014 & $-0.0009 * * *$ \\
\hline Mercedes 200E/E220 & $-0.0051 * *$ & $-0.0042 * * *$ & $-0.0029 * * *$ & $-0.0017 * * *$ & $-0.0026 * * *$ & $-0.0008 * * *$ \\
\hline Mercedes 320S/S350 & $\begin{array}{l}-0.0019 \\
\end{array}$ & $-0.0048 * * *$ & $-0.0035 * * *$ & $-0.0031 * * *$ & $-0.0072 * * *$ & $-0.0005 * * *$ \\
\hline Nissan Micra & -0.0033 & $-0.0033 * * *$ & $-0.0012 * * *$ & 0.0003 & $-0.0027 * * *$ & 0.0016 \\
\hline Nissan Sunny/Almera & -0.0005 & 0.0003 & 0.0001 & 0.0004 & $0.0022 *$ & -0.0002 \\
\hline Nissan Primera & $-0.0045 * *$ & -0.0002 & -0.0000 & 0.0007 & $0.0032 * * *$ & -0.0002 \\
\hline Opel Corsa & $-0.0048 * *$ & -0.0005 & $-0.0020 * * *$ & -0.0016 & $0.0055^{*}$ & $-0.0059 * *$ \\
\hline Opel Astra & -0.0012 & 0.0004 & $-0.0013^{* *}$ & $-0.0022 * *$ & 0.0028 & $-0.0075 * * *$ \\
\hline Opel Vectra & -0.0009 & 0.0000 & $-0.0017 * * *$ & $-0.0019 *$ & $0.0041^{*}$ & $-0.0062 * * *$ \\
\hline Opel Omega/Signum & $-0.0031 * *$ & -0.0002 & $-0.0013^{* *}$ & -0.0016 & 0.0031 & $-0.0075 * * *$ \\
\hline Peugeot 306/307 & $-0.0037 *$ & $-0.0017 * *$ & $-0.0013 * * *$ & $-0.0007 *$ & 0.0006 & $-0.0018 * * *$ \\
\hline Peugeot 405/406/407 & $-0.0041 *$ & -0.0006 & -0.0000 & 0.0004 & 0.0022 & -0.0061 \\
\hline Renault Clio & -0.0030 & 0.0002 & -0.0005 & -0.0003 & $0.0034 * * *$ & -0.0020 \\
\hline Renault 19/Megane & $\begin{array}{ll}-0.0038 \\
\end{array}$ & -0.0003 & -0.0006 & $\begin{array}{l}-0.0004 \\
\end{array}$ & $0.0024 * * *$ & $-0.0029 * * *$ \\
\hline Renault Laguna & $-0.0079 * * *$ & $-0.0025^{*}$ & $-0.0019 * * *$ & -0.008 & 0.0022 & -0.0012 \\
\hline Range Rover & -0.0107 & -0.0085 & $-0.0088 * * *$ & $-0.0126^{* * *}$ & -0.0246 & $-0.0032^{* *}$ \\
\hline Seat Ibiza & -0.0025 & -0.0007 & $-0.0012 * *$ & $-0.0021 * * *$ & -0.0022 & $-0.0013^{*}$ \\
\hline Seat Cordoba & $\begin{array}{l}-0.0039 \\
\end{array}$ & -0.0005 & -0.0007 & -0.0007 & 0.0006 & 0.0002 \\
\hline Seat Toledo & $-0.0058 * * *$ & $-0.0018 * *$ & $-0.0018 * * *$ & $-0.0013 * * *$ & 0.0009 & -0.0017 \\
\hline Toyota Starlet/Yaris & 0.0021 & -0.0030 & $-0.0020^{*}$ & $-0.0009 * *$ & $-0.0028 *$ & -0.0001 \\
\hline Toyota Corola & 0.0008 & -0.0006 & 0.0002 & 0.0004 & $-0.0017 * *$ & 0.0000 \\
\hline Toyota Carina/Avensis & -0.0007 & 0.0005 & -0.0002 & $-0.0008 *$ & 0.0007 & $-0.0021 * * *$ \\
\hline Volvo $440 / \mathrm{s} 40$ & -0.0028 & $-0.0022 * *$ & 0.0001 & 0.0017 & -0.0010 & 0.0011 \\
\hline VW Polo & $-0.0039 * *$ & $-0.0049 * * *$ & $-0.0038 * * *$ & $-0.0031 * * *$ & $-0.0053 * * *$ & $-0.0018 *$ \\
\hline VW Golf & -0.0014 & $-0.0017 * * *$ & $-0.0014 * * *$ & $-0.0014 * * *$ & $-0.0022 *$ & $-0.0014 * * *$ \\
\hline VW Passat & -0.0013 & $-0.0038 * * *$ & $-0.0028 * * *$ & $-0.0017 * * *$ & $-0.0031 * *$ & $\begin{array}{l}-0.0011 \\
\end{array}$ \\
\hline
\end{tabular}


Table 3

Trends in Price Dispersion: Sample group A

\begin{tabular}{|c|c|c|c|c|c|c|}
\hline Model & (1) & (2) & (3) & (4) & (5) & (6) \\
\hline & 1995-1998 & $1995-2001$ & $1995-2005$ & $1998-2005$ & $1998-2001$ & $2001-2005$ \\
\hline Alfa 145/147 & $0.0080^{*}$ & 0.0030 & -0.0006 & $-0.0049 * * *$ & $-0.0062 * *$ & $-0.0041 * * *$ \\
\hline Alfa $155 / 156$ & 0.0030 & 0.0011 & $-0.0033 * * *$ & $-0.0057 * * *$ & -0.0002 & -0.0066 \\
\hline Audi A4 & $-0.0031 * * *$ & $-0.0039 * * *$ & $-0.0045 * * *$ & $-0.0051 * * *$ & $-0.0054 * *$ & $-0.0036 * *$ \\
\hline Audi A6 & 0.0002 & $-0.0011 * *$ & $-0.0026 * * *$ & $-0.0039 * * *$ & -0.0014 & $-0.0077 * * *$ \\
\hline BMW 3-series & -0.0020 & $-0.0028 * * *$ & $-0.0030 * * *$ & $-0.0027 * * *$ & $-0.0015 * * *$ & $-0.0039 * * *$ \\
\hline BMW 5-series & -0.0013 & $-0.0020 * * *$ & $-0.0031 * * *$ & $-0.0036 * * *$ & $-0.0016^{*}$ & $-0.0056 * * *$ \\
\hline BMW 7-series & 0.0009 & -0.0002 & $-0.0011 * * *$ & $-0.0017 * * *$ & 0.0005 & $-0.0027 *$ \\
\hline Citroën $\mathrm{AX} / \mathrm{saxo} / \mathrm{C} 2$ & -0.0003 & 0.0015 & $-0.0016 * * *$ & $-0.0039 * * *$ & 0.0009 & $-0.0066 * * *$ \\
\hline Citroën ZX/ Xsara/C4 & 0.0013 & 0.0068 & 0.0004 & $-0.0039 *$ & $0.0091 * * *$ & $-0.0135 * * *$ \\
\hline Citroën Xantia/C5 & 0.0006 & $0.0070 * * *$ & 0.0014 & -0.0022 & 0.0076 & $-0.0036 * *$ \\
\hline Fiat Cinq./Seicento/Panda & $-0.0095 * * *$ & -0.0014 & $-0.0015^{*}$ & 0.0002 & 0.0067 & -0.0004 \\
\hline Fiat Punto & 0.0059 & 0.0001 & $-0.0018 *$ & $-0.0029 * *$ & -0.0021 & -0.0008 \\
\hline Fiat Bravo/Stilo & $-0.0032 * *$ & -0.0007 & -0.0010 & -0.0016 & -0.0017 & -0.0023 \\
\hline Ford Fiesta & 0.0003 & $-0.0024 * * *$ & $-0.0021 * * *$ & $-0.0021 * * *$ & $-0.0046 * * *$ & 0.0016 \\
\hline Ford Escort/Focus & -0.0043 & $-0.0041 * * *$ & $-0.0029 * * *$ & $-0.0017 *$ & -0.0019 & $-0.0042 *$ \\
\hline Ford Mondeo & -0.0025 & -0.0018 & $-0.0033 * * *$ & $-0.0043 * * *$ & -0.0007 & $-0.0090 * * *$ \\
\hline Honda Civic & 0.0008 & $-0.0048 * *$ & $-0.0028 * * *$ & -0.0019 & -0.0080 & -0.0008 \\
\hline Honda Accord & $-0.0083 * * *$ & -0.0030 & $-0.0024 * * *$ & -0.0015 & 0.0001 & $-0.0055^{* * *}$ \\
\hline Mazda 3-series & 0.0002 & -0.00002 & $-0.0022 * * *$ & $-0.0045 * * *$ & -0.0037 & -0.0036 \\
\hline Mercedes 180/c180 & $-0.0024 * * *$ & $-0.0024 * * *$ & $-0.0019 * * *$ & $-0.0017 * * *$ & $-0.0031 * * *$ & $-0.0007 * * *$ \\
\hline Mercedes 200E/E220 & -0.0010 & $-0.0017 * *$ & $-0.0014 * * *$ & $-0.0008 * * *$ & -0.0003 & $-0.0015 * * *$ \\
\hline Mercedes 320S/S350 & -0.0003 & $-0.0020 * * *$ & $-0.0012 * * *$ & -0.0005 & -0.0018 & 0.0001 \\
\hline Nissan Micra & $-0.0059 *$ & $-0.0047 * * *$ & $-0.0023 * * *$ & -0.0001 & -0.0020 & 0.0012 \\
\hline Nissan Sunny/Almera & -0.0062 & -0.0021 & $-0.0016 * * *$ & -0.0002 & 0.0032 & -0.0019 \\
\hline Nissan Primera & -0.0069 & 0.0009 & -0.0012 & -0.0010 & $0.0093 * * *$ & $-0.0067 * * *$ \\
\hline Opel Corsa & -0.0036 & -0.0008 & $-0.0027 * * *$ & $-0.0033 * * *$ & 0.0026 & $-0.0066 * * *$ \\
\hline Opel Astra & 0.0002 & 0.0001 & $-0.0012 *$ & $-0.0026 * * *$ & -0.0016 & -0.0033 \\
\hline Opel Vectra & 0.0009 & 0.0014 & $0.0025 * * *$ & $-0.0048 * * *$ & 0.0018 & -0.0061 \\
\hline Opel Omega/Signum & -0.0006 & -0.0007 & $-0.0026 * * *$ & $-0.0040 * * *$ & -0.0015 & $-0.0047 * * *$ \\
\hline Peugeot 306/307 & $0.0045 * * *$ & $0.0051^{* * *}$ & $0.0015^{*}$ & -0.0012 & 0.0045 & $-0.0065 * * *$ \\
\hline Peugeot 405/406/407 & 0.0046 & $0.0077 * *$ & $0.0045^{* * * *}$ & 0.0009 & 0.0059 & -0.0074 \\
\hline Renault Clio & 0.0006 & -0.0003 & $-0.0029 * * *$ & $-0.0049 * * *$ & -0.0010 & $-0.0079 * * *$ \\
\hline Renault 19/Megane & 0.0030 & 0.0004 & $-0.0023 * *$ & $-0.0044 * * *$ & -0.0005 & $-0.0080^{*}$ \\
\hline Renault Laguna & 0.0009 & $0.0014 *$ & $-0.0016 * * *$ & $-0.0039 * * *$ & 0.0004 & $-0.0056 * * *$ \\
\hline Range Rover & $-0.0075^{* *}$ & 0.0017 & -0.0024 & -0.0048 & 0.0042 & $-0.0032 *$ \\
\hline Seat Ibiza & $-0.0058 * * *$ & $-0.0026 * *$ & $-0.0044 * * *$ & $-0.0053 * * *$ & -0.0020 & -0.0040 \\
\hline Seat Cordoba & -0.0010 & -0.0016 & $-0.0044 * * *$ & $-0.0064 * * *$ & -0.0034 & $-0.0062 * * *$ \\
\hline Seat Toledo & $-0.0054 * *$ & $-0.0024 *$ & $-0.0028 * * *$ & $-0.0024 *$ & 0.0015 & $-0.0046 * * *$ \\
\hline Toyota Starlet/Yaris & -0.0052 & $-0.0028 * *$ & $-0.0023 * * *$ & $-0.0015 * *$ & -0.0002 & $-0.0034 * *$ \\
\hline Toyota Corola & $-0.0072 * * *$ & $-0.0047 * * *$ & $-0.0034 * * *$ & -0.0023 & -0.0013 & -0.0061 \\
\hline Toyota Carina/Avensis & -0.0019 & $-0.0017 * * *$ & $-0.0031 * * *$ & $-0.0043 * * *$ & $-0.0019 * * *$ & $-0.0071 * * *$ \\
\hline Volvo $440 / \mathrm{s} 40$ & -0.0077 & $-0.0045 * * *$ & $-0.0035 * * *$ & -0.0021 & 0.0005 & $-0.0068 * *$ \\
\hline VW Polo & -0.0017 & $-0.0014 * * *$ & $-0.0016 * * *$ & $-0.0017 * *$ & $-0.0020^{*}$ & -0.0005 \\
\hline VW Golf & 0.0027 & $0.0016^{*}$ & $0.0007 *$ & $-0.0004 *$ & -0.0007 & -0.0005 \\
\hline VW Passat & 0.0007 & $-0.0014 * *$ & $-0.0011 * * *$ & $-0.0010 * * *$ & $-0.0024 * *$ & -0.0002 \\
\hline
\end{tabular}


Table 4

Trends in Price Dispersion: Sample group B

\begin{tabular}{|c|c|c|c|c|c|c|}
\hline Model & (1) & (2) & (3) & (4) & $(5)$ & (6) \\
\hline & $1995-1998$ & $1995-2001$ & $1995-2005$ & $1998-2005$ & $1998-2001$ & $2001-2005$ \\
\hline Alfa 145/147 & $0.0142 *$ & 0.0049 & -0.0023 & $-0.0089 * * *$ & -0.0090 & $-0.0041 * *$ \\
\hline Alfa $155 / 156$ & $0.0128^{* *}$ & $0.0057 * * *$ & -0.0011 & $-0.0059 * * *$ & -0.0016 & -0.0051 \\
\hline Audi A4 & 0.0010 & $-0.0023 *$ & $-0.0035 * * *$ & $-0.0041 * * *$ & -0.0021 & $-0.0069 * * *$ \\
\hline Audi A6 & $0.0104 * *$ & -0.0014 & $-0.0024 * *$ & $-0.0043 * * *$ & $-0.0080 * *$ & $-0.0053 * *$ \\
\hline BMW 3-series & -0.0005 & 0.0004 & $-0.0015^{* *}$ & $-0.0028 * * *$ & 0.0002 & $-0.0038 * * *$ \\
\hline BMW 5-series & 0.0009 & -0.0018 & $-0.0031 * * *$ & $-0.0041 * * *$ & $-0.0032 *$ & $-0.0047 * * *$ \\
\hline BMW 7-series & -0.0075 & $-0.0099 * * *$ & $-0.0067 * * *$ & $-0.0041 * * *$ & $-0.0092 * * *$ & -0.0006 \\
\hline Citroën $\mathrm{AX} / \mathrm{saxo} / \mathrm{C} 2$ & $0.0059 *$ & 0.0018 & $-0.0027 *$ & $-0.0069 * * *$ & -0.0036 & -0.0093 \\
\hline Citroën ZX/ Xsara/C4 & $0.0098 * * *$ & $0.0039 *$ & -0.0008 & $-0.0058 * * *$ & -0.0045 & -0.0074 \\
\hline Citroën Xantia/C5 & $0.0042 *$ & $0.0030^{*}$ & -0.0010 & $-0.0045 * *$ & 0.0007 & $-0.0091 * *$ \\
\hline Fiat Cinq./Seicento/Panda & $0.0138^{*}$ & $0.0127 * * *$ & 0.0023 & $-0.0055^{*}$ & $0.0127^{* * *}$ & $-0.0168 * * *$ \\
\hline Fiat Punto & 0.0067 & $0.0056^{* *}$ & -0.0009 & $-0.0060 * * *$ & -0.0018 & -0.0024 \\
\hline Fiat Bravo/Stilo & $0.0213^{* * *}$ & $0.0077^{*}$ & -0.0009 & $-0.0098 * * *$ & -0.0095 & -0.0069 \\
\hline Ford Fiesta & 0.0086 & 0.0002 & $-0.0026^{*}$ & $-0.0049 * * *$ & -0.0045 & -0.0009 \\
\hline Ford Escort/Focus & 0.0088 & 0.0032 & -0.0008 & $-0.0041 * * *$ & -0.0017 & -0.0030 \\
\hline Ford Mondeo & $0.0114 * *$ & 0.0003 & $-0.0031 * * *$ & $-0.0069 * * *$ & $-0.0094 * * *$ & $-0.0044 * *$ \\
\hline Honda Civic & $0.0107 * * *$ & 0.0001 & $-0.0035 * * *$ & $-0.0088 * * *$ & $-0.0144 * * *$ & -0.0037 \\
\hline Honda Accord & $0.0079 * *$ & $0.0049 * * *$ & -0.0012 & $-0.0063 * * *$ & -0.0004 & $-0.0088 * * *$ \\
\hline Mazda 3-series & $0.0120 * * *$ & $0.0057^{*}$ & $-0.0036^{*}$ & $-0.0112 * * *$ & -0.0036 & $-0.0108 * * *$ \\
\hline Mercedes 180/c180 & -0.0026 & -0.0016 & $-0.0014 * * *$ & $-0.0011 * *$ & -0.0013 & -0.0005 \\
\hline Mercedes 200E/E220 & $-0.0086^{* * * *}$ & $-0.0040 * * *$ & $-0.0030 * * *$ & $-0.0016 * * *$ & 0.0000 & -0.0021 \\
\hline Mercedes 320S/S350 & -0.0048 & $-0.0047 * * *$ & $-0.0036^{* * *}$ & $-0.0033 * * *$ & $-0.0059 * *$ & $-0.0018^{*}$ \\
\hline Nissan Micra & 0.0020 & 0.0017 & -0.0012 & -0.0018 & $0.0067 * *$ & $-0.0073 * * *$ \\
\hline Nissan Sunny/Almera & $0.0111 * *$ & $0.0071 * * *$ & -0.0002 & $-0.0055 * * *$ & 0.0040 & $-0.0104 * * *$ \\
\hline Nissan Primera & 0.0055 & 0.0029 & -0.0009 & $-0.0034 * * *$ & 0.0015 & $-0.0045 * * *$ \\
\hline Opel Corsa & 0.0025 & 0.0007 & $-0.0037 * * *$ & $-0.0060 * * *$ & 0.0008 & $-0.0073 * *$ \\
\hline Opel Astra & $0.0060^{*}$ & 0.0024 & $-0.0020^{*}$ & $-0.0051 * * *$ & -0.0007 & $-0.0054 *$ \\
\hline Opel Vectra & 0.0060 & 0.0008 & $-0.0019^{* *}$ & $-0.0034 * * *$ & 0.0001 & $-0.0070 * * *$ \\
\hline Opel Omega/Signum & 0.0008 & $0.0025 * *$ & $-0.0022 * *$ & $-0.0054 * * *$ & 0.0021 & $-0.0105 * * *$ \\
\hline Peugeot 306/307 & $0.0127 * * *$ & $0.0063 * * *$ & -0.0012 & $-0.0071 * * *$ & 0.0010 & $-0.0112 * * *$ \\
\hline Peugeot 405/406/407 & 0.0019 & $0.0052 * * *$ & 0.0011 & -0.0012 & 0.0097 & $-0.0118 * * *$ \\
\hline Renault Clio & $0.0068 * *$ & $0.0049 * * *$ & 0.0004 & $-0.0032 * * *$ & 0.0004 & -0.0021 \\
\hline Renault 19/Megane & 0.0025 & 0.0023 & -0.0014 & $-0.0033 * *$ & 0.0033 & $-0.0057 * *$ \\
\hline Renault Laguna & 0.0019 & $0.0049 * *$ & -0.0014 & $-0.0053 * *$ & 0.0055 & $-0.0066^{*}$ \\
\hline Range Rover & -0.0068 & -0.0059 & $-0.0102 * * *$ & $-0.0185 * * *$ & -0.0313 & $-0.0047 * * *$ \\
\hline Seat Ibiza & $0.0087 *$ & 0.0000 & $-0.0021 * *$ & $-0.0056 * * *$ & $-0.0105 * * *$ & -0.0011 \\
\hline Seat Cordoba & $0.0100^{* *}$ & 0.0017 & -0.0016 & $-0.0052 * * *$ & $-0.0076 * *$ & 0.0003 \\
\hline Seat Toledo & $0.0113 * *$ & $0.0052 * *$ & -0.0008 & $-0.0060 * * *$ & -0.0029 & -0.0029 \\
\hline Toyota Starlet/Yaris & 0.0029 & -0.0035 & $-0.0047 * * *$ & $-0.0056^{* * *}$ & $-0.0071^{*}$ & $-0.0017 *$ \\
\hline Toyota Corola & 0.0073 & 0.0029 & -0.0010 & $-0.0050 * * *$ & -0.0046 & -0.0016 \\
\hline Toyota Carina/Avensis & $0.0050 *$ & $0.0036^{*}$ & -0.0017 & $-0.0065 * * *$ & -0.0030 & $-0.0068 * * *$ \\
\hline Volvo $440 / \mathrm{s} 40$ & 0.0025 & 0.0002 & 0.0010 & 0.0007 & -0.0039 & 0.0028 \\
\hline VW Polo & $0.0106 * * *$ & -0.0014 & $-0.0047 * * *$ & $-0.0081 * * *$ & $-0.0150 * * *$ & 0.0017 \\
\hline VW Golf & $0.0108 * * *$ & 0.0016 & $-0.0022 * *$ & $-0.0068 * * *$ & $-0.0096^{* * *}$ & $-0.0028 * *$ \\
\hline VW Passat & $0.0122 * * *$ & -0.0001 & $-0.0024 * *$ & $-0.0056 * * *$ & $-0.0108 * * *$ & -0.0006 \\
\hline
\end{tabular}

